\title{
Predictors of immunization coverage among 12-23 month old children in Ethiopia: systematic review and meta- analysis
}

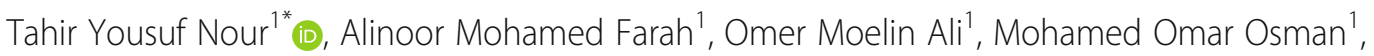
Mowlid Akil Aden ${ }^{1}$ and Kalkidan Hassen Abate $^{2}$

\begin{abstract}
Background: Immunization is one of modern medicine's greatest achievements in the last three decades. Annually it can prevent nearly 2 to 3 million deaths. Understanding the determinants of effective immunization coverage is a critical undertaking. Accordingly, we set out to check the best available evidence of outstanding predictors of immunization coverage among children aged 12-23 months in Ethiopia.

Method: Electronic databases including PubMed, Google Scholar, HINARI, and SCOPUS, Web of Science, African Journals Online, Ethiopian Medical Journals were searched. The search process, study selection, critical appraisal, and data extraction were done independently by two reviewers using Joanna Briggs Institute Meta-analysis for Review Instrument (JBI-MAStARI). The difference between reviewers was resolved with a third person. The risk of bias was assessed by the Newcastle Ottawa Tool for observational studies. Data were extracted using the Microsoft Excel checklist and exported to STATA 13. Heterogeneity was assessed using $I^{2}$, Funnel plot and Egger's test was used to check for publication bias.

Results: We identified 26 studies with 15,042 children with mothers/caretakers to assess factors associated with immunization coverage and significant factors were: maternal formal education, (OR=2.45; 95\% Cl: 1.62-3.72), paternal formal education, $(\mathrm{OR}=1.01 ; 95 \% \mathrm{Cl}: 0.27-3.77)$, residence, $(\mathrm{OR}=2.11 ; 95 \% \mathrm{Cl}: 1.00-4.45)$, birth at health facility (OR $=1.86 ; 95 \% \mathrm{Cl}: 0.99-3.49)$, family size less than four, $(\mathrm{OR}=1.81 ; 95 \% \mathrm{Cl}: 1.16-2.84)$, knowledge on age of immunization to be completed ( $\mathrm{OR}=6.18 ; 95 \% \mathrm{Cl}$ : 3.07-12.43), knowledge on immunization schedule ( $\mathrm{OR}=2.49$; 95\% Cl: 1.35-4.59), time to travel to health faculties, $(\mathrm{OR}=1.74 ; 95 \% \mathrm{Cl}: 0.62-4.89)$, antennal care, $(\mathrm{OR}=3.11 ; 95 \% \mathrm{Cl}$ : 1.64-5.88), and tetanus toxoid vaccination, ( $\mathrm{OR}=4.82 ; 95 \% \mathrm{Cl}: 2.99-7.75)$.

Conclusion: Our findings showed that literacy, residence, awareness, family size, maternal health services use, and proximity of the health facilities were factors associated with full immunization. This implies that there is a need for primary health service expansion and health education to "hard to reach areas" to improve immunization coverage for children aged 12-23 months.
\end{abstract}

Keywords: Systematic review, Meta-analysis, Ethiopia, Predictors of immunization coverage

\footnotetext{
* Correspondence: daahir571@gmail.com

'Department of public health, College of Medicine and Health Sciences, Jigjiga University, P.O. BOX 1020, Jigjiga, Ethiopia

Full list of author information is available at the end of the article
}

(c) The Author(s). 2020 Open Access This article is licensed under a Creative Commons Attribution 4.0 International License, which permits use, sharing, adaptation, distribution and reproduction in any medium or format, as long as you give appropriate credit to the original author(s) and the source, provide a link to the Creative Commons licence, and indicate if changes were made. The images or other third party material in this article are included in the article's Creative Commons licence, unless indicated otherwise in a credit line to the material. If material is not included in the article's Creative Commons licence and your intended use is not permitted by statutory regulation or exceeds the permitted use, you will need to obtain permission directly from the copyright holder. To view a copy of this licence, visit http://creativecommons.org/licenses/by/4.0/. The Creative Commons Public Domain Dedication waiver (http://creativecommons.org/publicdomain/zero/1.0/) applies to the data made available in this article, unless otherwise stated in a credit line to the data. 


\section{Background}

Immunization is a proven tool for controlling and eliminating life-threatening vaccine-preventable diseases and it averts 2 to 3 million deaths annually [1]. World Health Organization (WHO) launched an expanded program of immunization (EPI) in 1974 to all its members. The program aimed to eradicate vaccine-preventable diseases (VPDs) such as: diphtheria, measles, pertussis, tetanus, poliovirus, and tuberculosis [2].

A recent WHO report found that 13.5 million infants had not received a vaccination dose of any kind, 19.4 million did not recieve the third dose of DPT, and 12 million unvaccinated children lived in just ten countries: Angola, Brazil, the Democratic Republic of the Congo, Ethiopia, India, Indonesia, Nigeria, Pakistan, the Philippines, and Vietnam [3, 4].

Globally, from 1990 to 2018 under-five deaths ranged from 96 to 41 per 1000 live births. In the same years, Sub-Saharan Africa (SSA) ranged from (189 to 83/1000 live births). In 2018, under-five mortality in Ethiopia was (61 per 1000 live births) which is higher than most SSA countries [5].

The most common leading causes of under-five deaths are pneumonia, diarrhea, measles, and neonatal conditions that can be prevented or treated with simple affordable intervention such as vaccination or antibiotics [6]. Annually, 3.37 million children acquire pneumonia worldwide with an estimated 1.4 million or $18 \%$ of all under-five children deaths. The majority of children who acquired pneumonia live in South Asia and Sub-Saharan Africa [7]. In Ethiopia, 6.6\% of children under 5 had experienced symptoms of Acute Respiratory Infection (considered a proxy for pneumonia) and of those with the symptoms, treatment from a health facility or provider was sought for $31.6 \%$ only [8].

Universally, around 1.7 million cases of diarrhea occur annually [9]. In East African countries alone $13-32 \%$ of under-five mortality is due to diarrhea [10]. Only one in three children with episodes of diarrhea receive oral rehydration salts while less than $5 \%$ are receiving zinc supplement for diarrhea treatment [11]. Similarly, in Ethiopia $11.8 \%$ of under-five children experienced diarrhea and only $44.6 \%$ of children sought treatment from a health facility or health service provider. Among those children that sought treatment, $38 \%$ of children with diarrhea reportedly received rehydration solution in a form of an ORS sachet or a recommended home fluid [8].

Worldwide, 7 million people are affected by measles. Among the measles cases, $15 \%$ are severely complicated while only one-third of them received medical attention [12]. Likewise, measles incidence in Ethiopia is 50 cases per million per year which is way beyond the Ethiopian national measles elimination plan by the year 2020. The case fatality rate is 3 to $6 \%$ which is even underestimated due to incomplete reports [13].

Both community and morbidity based reports revealed that VPDs are public health problems in Ethiopia [14-16]. In Ethiopia, EPI was started in 1980 with aim of reducing maternal and child morbidity and mortality from VPDs [17]. EPI is provided through static, outreach, and mobile services with an immunization schedule of a dose bacillus Calmette-Guerin (BCG) and oral polio vaccine first dose (OPV0). Then three doses of OPV, three Penta-valent, two doses of Rota, and three pneumococcus vaccines which are given at 4th, 6th, 10th, and 14th weeks, respectively. Finally, the measles vaccine is given at the age of 9 months [18]. Recently, the second dose of measles vaccine was introduced to the routine immunization schedule and given at 15 months old children [13].

Ethiopian government has brought maternal, newborn, and child health as priority political agenda and maintained its commitment to improving the health and survival of women and children in the country (18). This has been demonstrated by massively expanding access to and utilization of key health care services through the Health Extension Program (HEP) (Tekelab et al., 2019). Health service delivery thus decentralized to nine regional states and two city councils, from zonal to Keble level (smallest administrative unit), with vaccines being supplied by the Federal Ministry of Health (FMOH) and developmental partners like GAVI and UNICEF, whom are also providing technical support to the government [18].

Although some studies reported determinants of immunization coverage in Ethiopia, they are not consistent and varied across the country. The reported determinants include ANC and PNC coverage, knowledge on age of a child to be vaccinated, parents formal education, place of delivery, residence, traveling time to health facilities, TT vaccination, family size, household wealth status, and marital status [19-23]. Thus, the current work aims at identifying relevant studies and summarizing major predictors of immunization coverage in Ethiopia. The results of this review will add to existing knowledge of the problem, and guide policymakers to improve immunization programs in Ethiopia.

\section{Method}

\section{Protocol and registration}

This systematic review and meta-analysis of predictors (factors) of immunization coverage among 12-23 month old children in Ethiopia was registered with international prospective register and systematic reviews PROSPERO 2020 RD42020166791. Available at: https://www.crd.york. ac.uk/prospero/display_record.php?ID=CRD42020166791. 


\section{Searching strategy}

We performed electronic searches of articles included in this systematic review from PubMed, Google Scholar, EMBASE, HINARI, SCOPUS, Web science, African Journals Online databases, and Ethiopian Medical Journal of which are all open sources. All relevant articles were also searched and retrieved manually from already identified articles' references. In addition, a Grey literature search was also done.

\section{Study selection}

The Preferred Systematic Reviews and Meta-Analysis (PRISMA) checklist was used in the formulation of systematic literature reviews. The reviewers searched articles from electronic databases based on their titles and abstracts. We selected studies assessed predictors of immunization coverage, Full text of selected studies reviewed, critical appraised, and data extracted. These search terms were pre-defined to allow a comprehensive search strategy that included all fields within records and Medical Subject Headings (MeSH) to expand the search in an advanced PubMed search as showed in Appendix I (Additional files 1).

\section{Inclusion and exclusion criteria}

\section{Study design and period}

observational study design (cohort, cross-sectional, and case-control) those reported predictors of immunization coverage among 12-23-month-old children published from 2009 were included.

\section{Study setting}

Both community and facility-based observational study designs were included.

\section{Population/participant}

Study participants were children aged 12-23 months.

Language: Studies reported in the English language only were considered to be eligible for this systematic review and meta-analysis.

\section{Publication condition}

Both published and unpublished articles were considered.

\section{Exposure}

Factors or determinants of immunization coverage in Ethiopia.

\section{Outcome}

Children aged 12-23 months who those are fully vaccinated.

\section{Exclusion}

After we have examined eligibility criteria of both published and unpublished studies, articles didn't fulfil inclusion criteria or full text were not got after solo authors contacted two times were excluded from this systematic review and meta-analysis. We also excluded case studies, reviews, theses, letters to editors, editorials, commentaries, and conference abstracts.

\section{Selection criteria}

\section{Measurement of the outcome variable}

The aim of this review is to assess predictors of immunization coverage among 12-23 months old children in Ethiopia. As per WHO definition, children are considered fully vaccinated when they receive one dose of Bacillus Calmette Guerin (BCG), three doses of DPT, three doses of Oral Polio Vaccine (OPV), and one dose of Measles Conjugated Vaccine (MCV) at the age of 9 to 12 months [24].

Identified predictors were maternal formal education, (formal education versus non-formal education), paternal formal education, (formal education and non-formal education), residence (urban versus rural), place of delivery (health facility versus home), family size $(<4$ and $>=$ $4)$, maternal knowledge on age to vaccinate their children (good versus poor), maternal knowledge on schedule (good versus verses poor), knowledge on benefit of vaccination (good versus poor), travelling time to health facility $(<=60 \mathrm{~min}$ and $>60 \mathrm{~min}$ ), antenatal care followup (yes versus no), postnatal care (yes versus no) and maternal TT vaccination (yes versus no).

\section{Data extraction procedures}

Two authors (TY and AM), independently extracted all necessary data based on pre-defined inclusion criteria using checklist ready from Microsoft Excel Sheet. Data extraction format consists: author, year of publication, region, study area, study design, sample size of determinant factors. Any disagreement between two authors was resolved through consensus between them, and a third reviewer (KHA) was asked to solve any disagreement that occurred. Data extraction format prepared was a two by two table in Appendex II (Additional file 2), $\log$ OR was calculated for each factor based on a two by two table from original studies.

\section{Quality assessment}

Two independent reviewers critically appraised articles with standardized critical appraisal format from Joanna Briggs Institute Meta-analysis for Review Instrument (JBI-MAStARI), Joanna Briggs Institute, University of Adelaide, Australia for observational studies (applied cross-sectional and case-control study designs). The checklist has eight questions for analytical cross- 
sectional and ten for case-control study designs with four possible answered (I. Yes, II. No, III. Unclear, IV. Not applicable) as shown (Appendix III) (Appendix IV). Three for cross-sectional studies i) selection of study design (four points). ii) comparability of study design (two points). iii) Outcome (three points). Three components for case-control i) selection of study (four points). ii) comparability of study design (two points). iii) ascertainment of exposure (three points) as showed (Additional files 3 and 4) respectively [25, 26]. Two reviewers also checked the quality of primary articles using Newcastle-Ottawa Quality Assessment Scale for observational studies (Additional file 5). Articles with methodological problems, incomplete, full texts not available were excluded from the final analysis.

\section{Data synthesis}

Data synthesis was carried out using STATA version 13. Forest plot was run and presented Odd Ratio (OR) with 95\% CI, $P$-value and $\mathrm{I}^{2}$. Heterogeneity was assessed based on (Cochrane handbook) with a cutoff point of $\mathrm{I}^{2}$ $0-40,30$ to $60 \%, 50$ to $90 \%, 75$ to $100 \%$ declared heterogeneity as: might not be important, moderate, substantial, and considerable heterogeneity. The funnel plot is the first step to assess the presence of publication bias and as a rule of thumb it is used only when metaanalysis contains ten and more studies [27]. Then Eggers' regression test was done to decide the presence of publication bias when $p$-value $<0.10$ [28]. This study was also reported based on the PRISMA flow diagram [29].

\section{Results}

In the first step, we searched, reviewed, and retrieved 383 published and unpublished articles from electronic databases. All articles were imported into EndNote software (version X7; Thomson Reuters, New York, NY) and 34 articles were excluded due to duplication. In addition, 291 articles were excluded for not relating to the topic, not done in Ethiopia, study design, and inconsistency with inclusion criteria set by the reviewers. Furthermore, 32 articles were excluded due to incorrect sample size, study design, and target. Finally, 26 articles were eligible and included in this meta-analysis as shown in the PRISMA follow chart (Fig. 1).

\section{Characteristics of the included studies}

All 26 included articles were observational studies (cross-sectional and case-control) with a total participant of 15,042 (Table 1). Their sample size ranged from 266 for Wonago district of the South region [37] to 1534 for Amibara woreda (district) of the Afar region [42]. Based on geographical distribution of the 26 included studies, eight studies were conducted in Amhara region [20, 22,
30, 32, 38, 43, 49, 50], nine in SNNP region [23, 33, 34, $36,37,40,41,46]$, four in Oromia region [21, 39, 48, 51], two from Tigray region [31, 35] one in Afar region [42], one in Somali region [44] and one from pastoral/ Semi-pastoral regions (Benishangul Gumuz, Gambella, Oromia, Somali, and Southern Nations, Nationalities and Peoples' regions) [47], and all were above cutoff points of $50 \%$. Based on factors associated with immunization coverage there were 12 selected factors and synthesized from identified eligible as shown (Table 2).

\section{Predictors of immunization coverage in Ethiopia Socio-demographic factors}

The result of this review revealed that some sociodemographic factors associated with immunization coverage among 12-23-month-old children in Ethiopia. These factors were; mother's educational status, father's educational status, residence, place of delivery and family size. Eight studies [23, 30, 33, 35, 41, 44, 48, 50] indicated that women's level of education was significantly associated with immunization coverage in Ethiopia. Women who had attended formal education were 2.45 times more likely to immunize their children compared to their counterpart $(\mathrm{OR}=2.45 ; 95 \% \mathrm{CI}: 1.62-3.72)$ (Fig. 2).

Three studies [32, 39, 41] also found father's educational status was associated with immunization coverage in Ethiopia. Children of fathers who had formal education were 1.01 more likely to complete routine vaccination than those who had non-formal education $(\mathrm{OR}=$ 0.01, 95\% CI: $0.27-3.77)$. Heterogeneity test for both women's and father's educational status was substantial and considerable with $\mathrm{I}^{2}=85.6$ and $94.3 \%$ respectively. Both of them showed there was no publication bias based on Egger's test with $p=0.883$ and $p=0.675$ respectively (Fig. 3). Six studies [20, 32, 33, 38, 44, 47] also showed that residence was significantly associated with immunization coverage in Ethiopia. This systematic review and meta-analysis revealed the rural residence was one of the determinants of immunization coverage. Women from rural residence were 1.58 times less likely to fully vaccinate their children compared to their counterparts $(\mathrm{OR}=2.11 ; 95 \% \mathrm{CI}: 1.00-4.45)$. There was no considerable heterogeneity among studies $\left(\mathrm{I}^{2}=94.6 \%\right) \mathrm{s}$ while Egger's test $p=0.784$ showed no significant publication bias (Fig. 4).

Similarly, nine studies [20, 21, 30, 33-35, 40, 41, 44] found that women who gave birth in the health facilities were 1.89 times more likely to complete routine immunization than those who gave birth at home (OR = 1.86; 95\% CI: 1.35-3.49). The heterogeneity test indicated that there was a considerable heterogeneity 


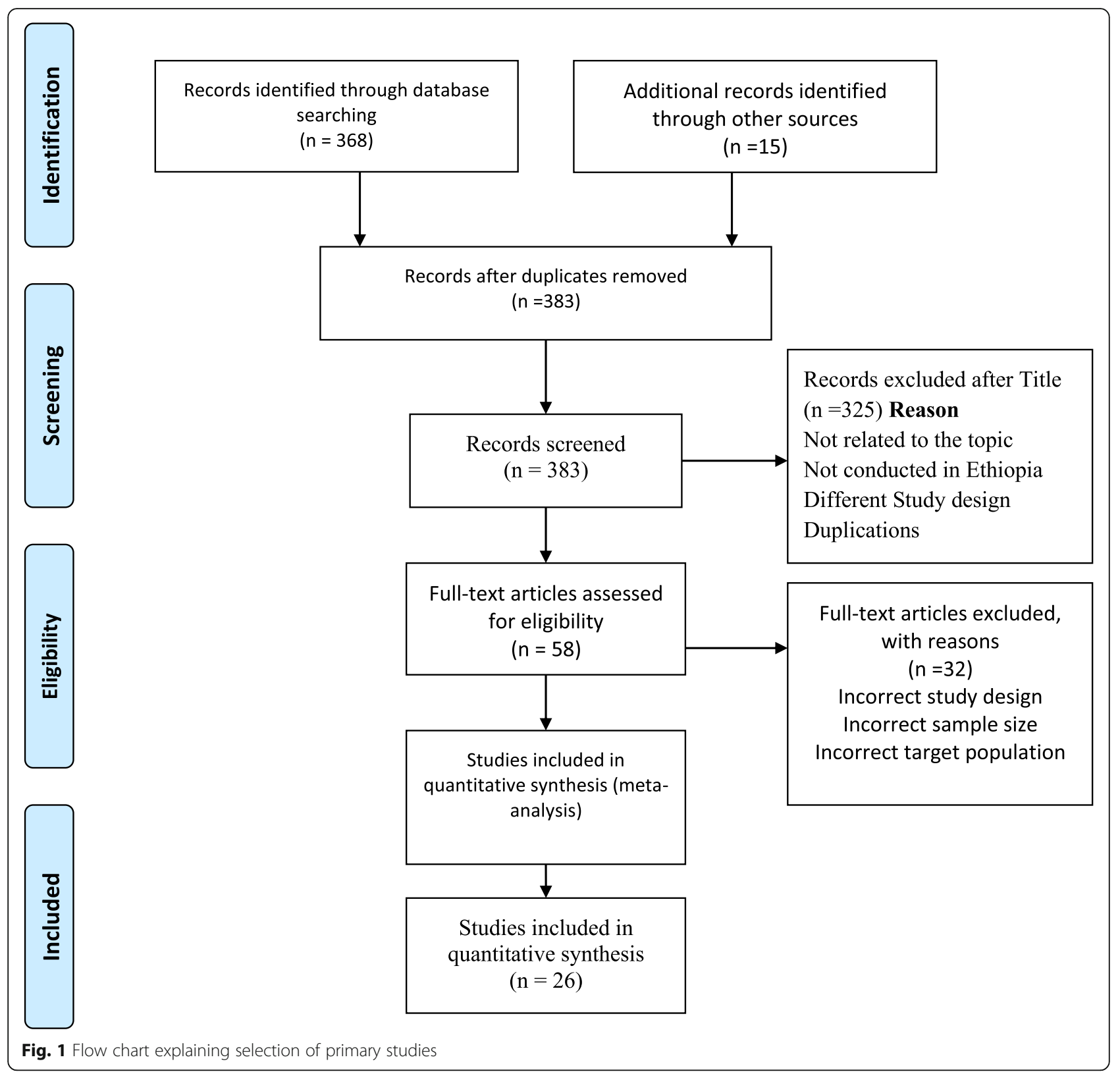

assessed place of delivery $\left(\mathrm{I}^{2}=94.6 \%\right)$ and no publication bias Egger's test was $p=0.428$ (Fig. 5).

Based on evidence from three studies [35, 45, 50] family size was associated with full vaccination. Households that had a family size less than four were more likely to complete immunization to their children compared to their counterpart $(\mathrm{OR}=1.81 ; 95 \% \mathrm{CI}: 1.16-2.84)$. There was a substantial heterogeneity test $\mathrm{I}^{2}=72.9 \%$, while Egger's test $p=0.767$ shows that there was no publication bias (Fig. 6).

\section{Maternal and health facility related factors}

Some maternal and health facility-related factors were also found to be significantly associated with immunization coverage. Maternal knowledge on age to complete immunization, knowledge on the immunization schedule, traveling time to the health facility was strongly associated with immunization coverage. Whereas, maternal knowledge on the benefit of immunization was not statistically significant to immunization coverage in Ethiopia.

Maternal knowledge on age to complete immunization was significantly associated with immunization coverage in Ethiopia [21, 38, 49, 52]. Women who had adequate knowledge on age to be completed routine immunization were more likely to vaccinate their children fully compared to women who had inadequate knowledge, $(\mathrm{OR}=6.18$; 95\% CI: 3.07-12.43). The heterogeneity test indicated that there was a considerable 
Table 1 Characteristics of included observational studies in this systematic review and meta-analysis in Ethiopia

\begin{tabular}{|c|c|c|c|c|c|c|c|c|c|}
\hline Author & Region & $\begin{array}{l}\text { Study } \\
\text { setting }\end{array}$ & Study area & $\begin{array}{l}\text { Study } \\
\text { design }\end{array}$ & $\begin{array}{l}\text { Sample } \\
\text { size }\end{array}$ & $\begin{array}{l}\text { Response } \\
\text { rate }\end{array}$ & NOS & $\begin{array}{l}\text { Study } \\
\text { subject }\end{array}$ & Predictors OR $95 \% \mathrm{Cl}$ \\
\hline $\begin{array}{l}\text { Animaw W } \\
\text { et al. } 2014 \\
\text { [30] }\end{array}$ & Amhara & $\begin{array}{l}\text { community } \\
\text { based }\end{array}$ & $\begin{array}{l}\text { Arba Minch town } \\
\text { and Arba Minch } \\
\text { Zuria District }\end{array}$ & CS & 630 & $100 \%$ & 7 & $\begin{array}{l}12-23 \\
\text { month }\end{array}$ & $\begin{array}{l}\text { Mother had formal education, }(\mathrm{OR}=4.60 ; 95 \% \mathrm{Cl} \text { : } \\
\text { 3.13-6.77), gave birth to the health facility, }(\mathrm{OR}= \\
3.20 \text {; } 95 \% \mathrm{Cl} \text { : } 2.00-5.15) \text {, knowledge on } \\
\text { immunization schedule (OR=0.46; } 95 \% \mathrm{Cl}: 0.30 \text {, } \\
\text { 0.71).travelling time < } 60 \mathrm{~min},(\mathrm{OR}=7.60 ; 95 \% \mathrm{Cl} \text { : } \\
\text { 2.67-21.66) }\end{array}$ \\
\hline $\begin{array}{l}\text { Aregawi G } \\
\text { et al. } 2017 \\
\text { [31] }\end{array}$ & Tigray & $\begin{array}{l}\text { community } \\
\text { based }\end{array}$ & $\begin{array}{l}\text { Laelay Adiabo } \\
\text { District }\end{array}$ & UMCC & 270 & $100 \%$ & 8 & $\begin{array}{l}12-23 \\
\text { month }\end{array}$ & $\begin{array}{l}\text { Knowledge on schedule, }(\mathrm{OR}=6.98 ; 95 \% \mathrm{Cl}: 3.94- \\
\text { 12.37), PNC follow up, }(\mathrm{OR}=7.08 ; 95 \% \mathrm{Cl}: 3.67- \\
13.65)\end{array}$ \\
\hline $\begin{array}{l}\text { Asfaw A } \\
\text { et al. } 2019 \\
\text { [23] }\end{array}$ & SNNP & $\begin{array}{l}\text { community } \\
\text { based }\end{array}$ & $\begin{array}{l}\text { Sodo Zurea } \\
\text { Distric }\end{array}$ & UMCC & 344 & $100 \%$ & 9 & $\begin{array}{l}12-23 \\
\text { month }\end{array}$ & $\begin{array}{l}\text { Had formal education, }(\mathrm{OR}=2.34 ; 95 \% \mathrm{Cl}: 1.15- \\
3.64), \text { knowledge on immunization schedule, } \\
(\mathrm{OR}=2.79,95 \% \mathrm{Cl}: 1.73-4.48), \text { PNC follow up, } \\
(\mathrm{OR}=2.69 ; 95 \% \mathrm{Cl}: 1.73-4.48)\end{array}$ \\
\hline $\begin{array}{l}\text { Ayal D and } \\
\text { Bekele T } \\
2014 \text { [32] }\end{array}$ & Amhara & $\begin{array}{l}\text { community } \\
\text { based }\end{array}$ & Mecha District & CS & 497 & $100 \%$ & 9 & $\begin{array}{l}12-23 \\
\text { month }\end{array}$ & $\begin{array}{l}\text { Father had formal education, }(\mathrm{OR}=1.28,95 \% \mathrm{Cl} \text { : } \\
0.90,1.82) \text {, urban residence, }(\mathrm{OR}=3.94,95 \% \mathrm{Cl} \text { : } \\
1.76-8.82) \text {. gave birth to the health facility, }(\mathrm{OR}= \\
1.92,95 \% \mathrm{Cl}: 1.30,2.84) \text {, knowledge on } \\
\text { immunization schedule, (OR }=2.77,95 \% \mathrm{Cl}: 1.42- \\
5.39)\end{array}$ \\
\hline $\begin{array}{l}\text { Birhan, Y., } \\
\text { et al. } 2014 \\
\text { [33] }\end{array}$ & SNNP & $\begin{array}{l}\text { community } \\
\text { based }\end{array}$ & $\begin{array}{l}\text { Hawassa Zuria } \\
\text { District }\end{array}$ & UMCC & 308 & $98 \%$ & 8 & $\begin{array}{l}12-23 \\
\text { month }\end{array}$ & 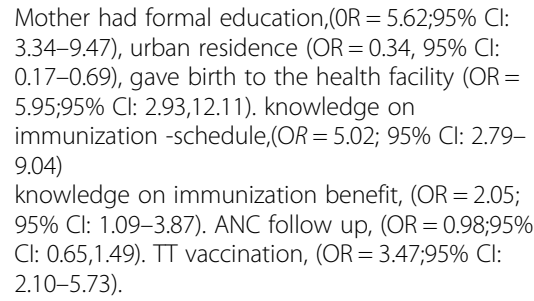 \\
\hline
\end{tabular}

\begin{tabular}{|c|c|c|c|c|c|c|c|c|c|}
\hline $\begin{array}{l}\text { Bizuneh } \\
\text { Ayano } 2014 \\
{[34]}\end{array}$ & SNNP & $\begin{array}{l}\text { community } \\
\text { based }\end{array}$ & Hosanna Town & CS & 508 & $100 \%$ & 8 & $\begin{array}{l}12-23 \\
\text { month }\end{array}$ & $\begin{array}{l}\text { knowledge on immunization schedule, }(\mathrm{OR}=2.28 \text {; } \\
\text { 95\% Cl:1.79-9.04). ANC follow up, (OR }=6.49 ; 95 \% \\
\text { Cl: } 2.76-15.26) \text {. TT vaccination, (OR }=4.85 ; 95 \% \mathrm{Cl} \text { : } \\
\text { 3.38-6.97) }\end{array}$ \\
\hline $\begin{array}{l}\text { Etana and } \\
\text { Deressa } \\
2012 \text { [21] }\end{array}$ & Oromia & $\begin{array}{l}\text { community } \\
\text { based }\end{array}$ & Ambo District & CS & 536 & $100 \%$ & 7 & $\begin{array}{l}12-23 \\
\text { month }\end{array}$ & $\begin{array}{l}\text { Gave birth to the health facility, }(\mathrm{OR}=0.71 ; 95 \% \mathrm{Cl} \text { : } \\
0.43,1.17) \text {. knowledge on age immunization } \\
\text { complete, }(\mathrm{OR}=10.03 ; 95 \% \mathrm{Cl}: 5.02,9.75) \text {. ANC } \\
\text { follow up, }(\mathrm{OR}=6.38 ; 95 \% \mathrm{Cl}: 4.01,10.15)\end{array}$ \\
\hline $\begin{array}{l}\text { Girmay and } \\
\text { Dadi } 2019 \\
\text { [35] }\end{array}$ & Tigray & $\begin{array}{l}\text { community } \\
\text { based }\end{array}$ & $\begin{array}{l}\text { Debre Markos } \\
\text { Town }\end{array}$ & CS & 620 & $99.5 \%$ & 8 & $\begin{array}{l}12-23 \\
\text { month }\end{array}$ & $\begin{array}{l}\text { Mother had formal education, }(\mathrm{OR}=1.77 ; 95 \% \mathrm{Cl} \text { : } \\
1.20-2.59) \text {. gave birth to the health facility, }(\mathrm{OR}= \\
3.52 ; 95 \% \mathrm{Cl}: 2.36-5.25) \text {, family size }>=4 \text {, }(\mathrm{OR}= \\
2.34 ; 95 \% \mathrm{Cl}: 1.54,3.57) \text {. knowledge on } \\
\text { immunization schedule, (OR }=0.22 ; 95 \% \mathrm{Cl}: 0.15- \\
0.34) \text {, travelling time }<60 \text { min, }(\mathrm{OR}=2.20 ; 95 \% \mathrm{Cl} \text { : } \\
1.47-3.30)\end{array}$ \\
\hline $\begin{array}{l}\text { Gualu and } \\
\text { Dilia } 2017 \\
\text { [22] }\end{array}$ & Amhara & $\begin{array}{l}\text { community } \\
\text { based }\end{array}$ & $\begin{array}{l}\text { Debre Markos } \\
\text { Town }\end{array}$ & CS & 288 & $96.8 \%$ & 7 & $\begin{array}{l}12-23 \\
\text { month }\end{array}$ & ANC follow up, $(\mathrm{OR}=3.67 ; 95 \% \mathrm{Cl}: 1.32-10.19)$ \\
\hline $\begin{array}{l}\text { Hailu et al. } \\
2019 \text { [36] }\end{array}$ & SNNP & $\begin{array}{l}\text { community } \\
\text { based }\end{array}$ & Wonago district & CS & 1116 & $82.70 \%$ & 7 & $\begin{array}{l}12-23 \\
\text { month }\end{array}$ & $\begin{array}{l}\text { Knowledge on immunization schedule, }(\mathrm{OR}=2.57 \text {; } \\
\text { 95\% Cl: } 2.00-3.30) \text {. ANC follow up, }(\mathrm{OR}=0.61 ; 95 \% \\
\mathrm{Cl}: 0.46-0.80), \pi \text { vaccination, }(\mathrm{OR}=3.37 ; 95 \% \mathrm{Cl} \text { : } \\
2.58-4.40)\end{array}$ \\
\hline $\begin{array}{l}\text { Henok T } \\
\text { et al. } 2009 \\
\text { [37] }\end{array}$ & SNNP & $\begin{array}{l}\text { community } \\
\text { based }\end{array}$ & Wonago district & UMCC & 266 & $99.2 \%$ & 7 & $\begin{array}{l}12-23 \\
\text { month }\end{array}$ & $\begin{array}{l}\text { Knowledge on immunization benefit, }(\mathrm{OR}=2.58 \text {; } \\
95 \% \mathrm{Cl} \text { : } 1.09-6.15) \text {. }\end{array}$ \\
\hline $\begin{array}{l}\text { Kassahun } \\
\text { et al. } 2015 \\
\text { [38] }\end{array}$ & Amhara & $\begin{array}{l}\text { community } \\
\text { based }\end{array}$ & $\begin{array}{l}\text { Lay Armacheho } \\
\text { district }\end{array}$ & CS & 751 & $99.2 \%$ & 8 & $\begin{array}{l}12-23 \\
\text { month }\end{array}$ & $\begin{array}{l}\text { Knowledge on age immunization complete, } \\
(\mathrm{OR}=2.42,95 \% \mathrm{Cl}: 1.71-3.43) \text {, urban residence, } \\
(\mathrm{OR}=1.47 ; 95 \% \mathrm{Cl}: 0.96-2.26) \\
\text { TT vaccination (OR }=2.45 ; 95 \% \mathrm{Cl}: 1.64-3.64)\end{array}$ \\
\hline $\begin{array}{l}\text { Lake et al. } \\
2014[36]\end{array}$ & Amhra & $\begin{array}{l}\text { community } \\
\text { based }\end{array}$ & Dassie town & CS & 724 & $100 \%$ & 7 & $\begin{array}{l}12-23 \\
\text { month }\end{array}$ & $\begin{array}{l}\text { Knowledge on immunization schedule, }(\mathrm{OR}=6.12 \text {, } \\
95 \% \mathrm{Cl}: 4.41,8.49) \text {. knowledge on age } \\
\text { immunization complete, (OR }=0.56 ; 95 \% \mathrm{Cl}: 0.24- \\
1.29) \\
\text { family size }>=4 \text {, (OR }=1.14 ; 95 \% \mathrm{Cl}: 0.75-1.72)\end{array}$ \\
\hline Legesse and & Oromia & community & Sinana district & CS & 519 & $98.5 \%$ & 8 & $12-23$ & Knowledge on immunization schedule, $(\mathrm{OR}=2.16$ \\
\hline
\end{tabular}


Table 1 Characteristics of included observational studies in this systematic review and meta-analysis in Ethiopia (Continued)

\begin{tabular}{|c|c|c|c|c|c|c|c|c|c|}
\hline Author & Region & $\begin{array}{l}\text { Study } \\
\text { setting }\end{array}$ & Study area & $\begin{array}{l}\text { Study } \\
\text { design }\end{array}$ & $\begin{array}{l}\text { Sample } \\
\text { size }\end{array}$ & $\begin{array}{l}\text { Response } \\
\text { rate }\end{array}$ & NOS & $\begin{array}{l}\text { Study } \\
\text { subject }\end{array}$ & Predictors OR 95\% Cl \\
\hline $\begin{array}{l}\text { Dechasa } \\
2015[39]\end{array}$ & & based & & & & & & month & $\begin{array}{l}\text { 95\% Cl: } 1.45-3.22) \text {, father had formal education, } \\
(\mathrm{OR}=0.18 ; 95 \% \mathrm{Cl}: 0.07-0.44) \text {, travelling time }<60 \\
\mathrm{~min},(\mathrm{OR}=2.16 ; 95 \% \mathrm{Cl}: 0.69-6.72), \\
\text { ANC follow up, (OR }=3.03,95 \% \mathrm{Cl}: 2.02-4.55)\end{array}$ \\
\hline $\begin{array}{l}\text { Melaku } \\
\text { Kindie et al. } \\
2018[40]\end{array}$ & SNNP & $\begin{array}{l}\text { community } \\
\text { based }\end{array}$ & Amanuel district & UMCC & 308 & $100 \%$ & 9 & $\begin{array}{l}12-23 \\
\text { month }\end{array}$ & $\begin{array}{l}\text { gave birth to the health facility, }(\mathrm{OR}=0.15 ; 95 \% \mathrm{Cl} \text { : } \\
0.09-0.25) \text {, ANC follow up, }(\mathrm{OR}=5.10 ; 95 \% \mathrm{Cl}: 3.12- \\
\text { 8.34), PNC follow up, }(\mathrm{OR}=3.28 ; 95 \% \mathrm{Cl}: 1.84-5.85) \text {. }\end{array}$ \\
\hline $\begin{array}{l}\text { Meleko et al. } \\
2018[41]\end{array}$ & SNNP & $\begin{array}{l}\text { community } \\
\text { based }\end{array}$ & $\begin{array}{l}\text { Mizan Aman } \\
\text { town }\end{array}$ & $\mathrm{CC}$ & 322 & $100 \%$ & 8 & $\begin{array}{l}12-23 \\
\text { month }\end{array}$ & $\begin{array}{l}\text { Mother had formal education, }(\mathrm{OR}=3.03 ; 95 \% \mathrm{Cl} \text { : } \\
1.91-4.80) \text {, gave birth to the health facility, }(\mathrm{OR}= \\
2.05 ; 95 \% \mathrm{Cl}: 1.30-3.23)\end{array}$ \\
\hline $\begin{array}{l}\text { Mebrahtom } \\
\text { and Birhane } \\
2013 \text { [42] }\end{array}$ & Afar & $\begin{array}{l}\text { community } \\
\text { based }\end{array}$ & Amibara district & CS & 1534 & $98.3 \%$ & 8 & $\begin{array}{l}12-23 \\
\text { month }\end{array}$ & $\begin{array}{l}\text { Knowledge on immunization schedule, } 21.50 \\
\text { (11.47-40.30). ANC follow up, }(\mathrm{OR}=20.49 ; 95 \% \mathrm{Cl} \text { : } \\
\text { 10.32-40.70), PNC follow up, (OR=4.07;95\% Cl: } \\
\text { 2.67,6.22). Tा vaccination, (OR }=36.80 ; 95 \% \mathrm{Cl} \text { : } \\
\text { 14.95-90.56) }\end{array}$ \\
\hline $\begin{array}{l}\text { Mekonnen } \\
\text { et al. } 2019 \\
\text { [43] }\end{array}$ & Amhara & $\begin{array}{l}\text { community } \\
\text { based }\end{array}$ & $\begin{array}{l}\text { Minjarshenkora } \\
\text { district }\end{array}$ & CS & 566 & $98.8 \%$ & 8 & $\begin{array}{l}12-23 \\
\text { month }\end{array}$ & $\begin{array}{l}\text { Travelling time < } 60 \mathrm{~min} \text {, }(\mathrm{OR}=1.95 ; 95 \% \mathrm{Cl}: 1.30- \\
\text { 2.92) }\end{array}$ \\
\hline $\begin{array}{l}\text { Mohammed } \\
\text { and Atomsa } \\
203\end{array}$ & Oromia & $\begin{array}{l}\text { community } \\
\text { based }\end{array}$ & $\begin{array}{l}\text { Kombolcha } \\
\text { District }\end{array}$ & CS & 685 & $98.7 \%$ & 7 & $\begin{array}{l}12-23 \\
\text { month }\end{array}$ & ANC follow up, (OR = 2.42;95\% Cl: 1.64-3.58) \\
\hline $\begin{array}{l}\text { Mohamud } \\
\text { et al. } 2014 \\
\text { [44] }\end{array}$ & Somali & $\begin{array}{l}\text { community } \\
\text { based }\end{array}$ & Jigjig district & CS & 582 & $100 \%$ & 8 & $\begin{array}{l}12-23 \\
\text { month }\end{array}$ & $\begin{array}{l}\text { Mother had formal education, }(\mathrm{OR}=3.63 ; 95 \% \mathrm{Cl} \text { : } \\
2.17-6.08) \text {, gave birth to the health facility, }(\mathrm{OR}= \\
3.06 ; 95 \% \mathrm{Cl}: 2.10-4.47) \text {, urban residence, }(\mathrm{OR}=2.63 \text {; } \\
95 \% \mathrm{Cl} \text { : 1.85-3.73), } T \text { ' vaccination, (OR }=4.94 ; 95 \% \\
\mathrm{Cl}: 2.82-8.66)\end{array}$ \\
\hline $\begin{array}{l}\text { Negussie A } \\
\text { et al. } 2016 \\
\text { [45] }\end{array}$ & SNNP & $\begin{array}{l}\text { community } \\
\text { based }\end{array}$ & Arbegona district & CS & 548 & $99.45 \%$ & 7 & $\begin{array}{l}12-23 \\
\text { month }\end{array}$ & $\begin{array}{l}\text { Knowledge on immunization benefit, }(\mathrm{OR}=6.30 \text {; } \\
95 \% \text { Cl: } 2.33-3.87) \text {. family size }>=4 \text {, }(\mathrm{OR}=2.22 \text {; } \\
95 \% \text { Cl: } 1.52-3.24)\end{array}$ \\
\hline $\begin{array}{l}\text { Tefera et al. } \\
2018[46]\end{array}$ & SNNP & $\begin{array}{l}\text { community } \\
\text { based }\end{array}$ & Worabe town & CS & 484 & $89.6 \%$ & 8 & $\begin{array}{l}12-23 \\
\text { month }\end{array}$ & $\begin{array}{l}\text { travelling time }<60 \mathrm{~min},(\mathrm{OR}=0.30 ; 95 \% \mathrm{Cl}: 0.20- \\
0.44) \text {, ANC follow up, (OR=0.32;95\% Cl: } 0.11-0.95)\end{array}$ \\
\hline $\begin{array}{l}\text { Tesfaye et al. } \\
2018[20]\end{array}$ & Amhara & $\begin{array}{l}\text { community } \\
\text { based }\end{array}$ & East Gojam Zone & CS & 846 & $98.1 \%$ & 8 & $\begin{array}{l}12-23 \\
\text { month }\end{array}$ & $\begin{array}{l}\text { Urban Residence, }(\mathrm{OR}=1.63 ; 95 \% \mathrm{Cl}: 1.23-2.15) \text {, } \\
\text { ANC follow up, }(\mathrm{OR}=3.86 ; 95 \% \mathrm{Cl}: 2.42-6.16)\end{array}$ \\
\hline $\begin{array}{l}\text { Tessema } \\
\text { et al. } 2019 \\
{[47]}\end{array}$ & $\begin{array}{l}\text { Pastoral/ } \\
\text { semi- } \\
\text { pastoral }\end{array}$ & $\begin{array}{l}\text { community } \\
\text { based }\end{array}$ & $\begin{array}{l}\text { CORE Group Polio } \\
\text { Project } \\
\text { implementation } \\
\text { areas }\end{array}$ & CS & 600 & $96.6 \%$ & 8 & $\begin{array}{l}12-23 \\
\text { month }\end{array}$ & Urban residence, $(\mathrm{OR}=9.39 ; 95 \% \mathrm{Cl}: 6.42-13.75)$ \\
\hline $\begin{array}{l}\text { Wado et al. } \\
2014[48]\end{array}$ & Oromia & $\begin{array}{l}\text { community } \\
\text { based }\end{array}$ & $\begin{array}{l}\text { Gilgel Gibe Health } \\
\text { and Demographic } \\
\text { Surveillance } \\
\text { System }\end{array}$ & CS & 889 & $100 \%$ & 8 & $\begin{array}{l}12-23 \\
\text { month }\end{array}$ & $\begin{array}{l}\text { Mother had formal education, }(\mathrm{OR}=1.46 ; 95 \% \mathrm{Cl} \text { : } \\
1.07-1.99)\end{array}$ \\
\hline $\begin{array}{l}\text { Yismaw A } \\
\text { et al. } 2019 \\
\text { [49] }\end{array}$ & Amhara & $\begin{array}{l}\text { community } \\
\text { based }\end{array}$ & Gondar city & CS & 301 & $100 \%$ & 8 & $\begin{array}{l}12-23 \\
\text { month }\end{array}$ & $\begin{array}{l}\text { Knowledge on age immunization complete, }(\mathrm{O} R= \\
9.43 ; 95 \% \text { Cl: } 5.16-17.23) \text {, knowledge on } \\
\text { immunization benefit, }(\mathrm{OR}=1.54 ; 95 \% \mathrm{Cl}: 0.82- \\
\text { 2.89) }\end{array}$ \\
\hline
\end{tabular}

Key: CS cross-sectional, UMCC unmatched case-control

significant heterogeneity $\left(\mathrm{I}^{2}=90.2 \%\right)$, and Egger's test found there was no publication bias $(p=0.432)$ (Fig. 7).

Furthermore, 12 studies [20, 23, 30-36, 39, 42, 50] showed maternal knowledge on immunization schedule was significantly associated with vaccination coverage in Ethiopia. Women who had good knowledge of immunization schedules were 2.49 times more likely to fully vaccinate their children compared to women who had poor knowledge of the immunization schedule, (OR, 2.49; 95\% CI: $1.35-4.59)$. There was a considerable heterogeneity test among studies $\left(\mathrm{I}^{2}=96.2 \%\right)$ as shown (Fig. 8). Funnel plot illustrated the presence of publication bias but Egger's test revealed there was no publication bias (0.621) and we concluded that there was no publication bias as shown (Fig. 9). On the other hand, four studies [33, 37, 40,49] revealed that there were no significant association between knowledge on the benefit of immunization and immunization coverage (Fig. 10). Six studies [30, 35, 39, 43, 46, 53] also indicated there was an association between traveling time to the health facility and immunization coverage. Women who had to walk for less than or equal to $60 \mathrm{~min}$ to the health facility were more likely to fully vaccinate their children compared to those who had more than a $1 \mathrm{~h}$ walk (OR, 
Table 2 Meta-analysis summary of included and synthesised predictors on immunization coverage in Ethiopia

\begin{tabular}{|c|c|c|c|c|c|c|}
\hline s.no & Factors /authors & $\begin{array}{l}\text { Number of } \\
\text { study }\end{array}$ & OR, $95 \% \mathrm{Cl}$ & $\begin{array}{l}P- \\
\text { value }\end{array}$ & $1^{2}$ & $\begin{array}{l}\text { Egger's test ( } p \text { - } \\
\text { value) }\end{array}$ \\
\hline 1 & $\begin{array}{l}\text { Mother with formal education }[23,30,33,35,41,44,48 \text {, } \\
50]\end{array}$ & 8 & $\begin{array}{l}(\mathrm{OR}=2.45 ; 95 \% \mathrm{Cl}: 1.62- \\
3.72)\end{array}$ & 0.000 & $85.6 \%$ & 0.883 \\
\hline 2 & Father with formal education $[32,39,41]$ & 3 & $\begin{array}{l}(\mathrm{OR}=1.01 ; 95 \% \mathrm{Cl}: 0.27- \\
0.77)\end{array}$ & 0.000 & $94.5 \%$ & 0.675 \\
\hline 3 & Residence $[20,32,33,38,44,47]$ & 6 & $\begin{array}{l}(\mathrm{OR}=2.11 ; 95 \% \mathrm{Cl}: 1.00- \\
4.45)\end{array}$ & 0.000 & $94.6 \%$ & 0.784 \\
\hline 4 & $\begin{array}{l}\text { Place of delivery (health facility) }[20,21,30,33-35,40,41 \text {, } \\
44]\end{array}$ & 9 & $\begin{array}{l}(\mathrm{OR}=1.86 ; 95 \% \mathrm{Cl}: 0.99- \\
3.49)\end{array}$ & 0.000 & $94.6 \%$ & 0.428 \\
\hline 5 & Family size $>=4[35,45,50]$ & 3 & $\begin{array}{l}(\mathrm{OR}=1.81 ; 95 \% \mathrm{Cl}: 1.16- \\
2.84)\end{array}$ & 0.026 & $72.9 \%$ & 0.767 \\
\hline 6 & knowledge on age to complete $[21,38,49,52]$ & 4 & $\begin{array}{l}(\mathrm{OR}=6.18 ; 95 \% \mathrm{Cl}: 3.07- \\
12.43)\end{array}$ & 0.000 & $90.2 \%$ & 0.432 \\
\hline 7 & Knowledge on schedule $[20,23,30-36,39,42,50]$ & 12 & $\begin{array}{l}(\mathrm{OR}=2.49 ; 95 \% \mathrm{Cl}: 1.35- \\
4.59)\end{array}$ & 0.000 & $96.2 \%$ & 0.621 \\
\hline 8 & Knowledge on benefit of immunization $[33,37,40,49]$ & 4 & $\begin{array}{l}(\mathrm{OR}=2.43 ; 95 \% \mathrm{Cl}: 1.44- \\
4.09)\end{array}$ & 0.129 & $47.1 \%$ & 0.091 \\
\hline 9 & $\begin{array}{l}\text { Travelling time to health facilities }>=60 \mathrm{~min}[30,35,39 \text {, } \\
43,46,53]\end{array}$ & 6 & $\begin{array}{l}(\mathrm{OR}=1.76 ; 95 \% \mathrm{Cl}: 0.62- \\
4.89)\end{array}$ & 0.000 & $94.5 \%$ & 0.535 \\
\hline 10 & ANC follow up $[20-22,33-36,39,40,42,46,51]$ & 12 & $\begin{array}{l}(\mathrm{OR}=3.11 ; 95 \% \mathrm{Cl}: 1.64- \\
5.88)\end{array}$ & 0.000 & $95.0 \%$ & 0.104 \\
\hline 11 & PNC follow up $[23,31,40,42]$ & 4 & $\begin{array}{l}(\mathrm{OR}=3.83 ; 95 \% \mathrm{Cl}: 2.65- \\
5.52)\end{array}$ & 0.105 & $51.2 \%$ & 0.405 \\
\hline 12 & $\pi$ vaccination $[33,34,36,38,42,44]$ & 5 & $\begin{array}{l}(\mathrm{OR}=4.84 ; 95 \% \mathrm{Cl}: 2.99- \\
7.75)\end{array}$ & 0.000 & $85.8 \%$ & 0.124 \\
\hline
\end{tabular}

2.33; 95\% CI: 0.80-6.79). Heterogeneity test ( $\left.\mathrm{I}^{2}=95.9 \%\right)$ showed there was no considerable heterogeneity. Based on Egger's test weighted regression statistics $(p=0.535)$ there was no indicative publication bias (Fig. 11).

\section{Maternal health care utilization factors}

Maternal health care utilization factors such as antenatal care, maternal TT vaccination was also significantly associated while postnatal care was not significantly associated with immunization coverage in Ethiopia. Twelve studies [20-22, 33-36, 39, 40, 42, 46, 51] found a significant association between antenatal care and full vaccination in Ethiopia. Women who had ANC follow up were three times more likely to complete their children's vaccination program compared to women who followed ANC, $(\mathrm{OR}=3.11$; 95\% CI: 1.64-5.88) (Fig. 12). With the random effect model $\mathrm{I}^{2}=95.0 \%$ substantial heterogeneity was found. Funnel plot was asymmetrical which indicates the presence of publication bias while Egger's test $(p=0.104)$ indicated the absence of publication bias, thus we concluded that there was no publication bias (Fig. 13). On the contrary, four studies [23, 31, 40, 42] revealed that there were no association between postnatal care and immunization coverage in Ethiopia, as it is depicted (Fig. 14). Six studies [33, 34, 36, 38, 42, 44] also indicated that TT vaccination was significantly associated with immunization coverage. Women who took TT vaccination during ANC follow-up were 4.82 times more likely to complete immunization of their children compared to those who had not taken TT vaccination $(\mathrm{OR}=4.82 ; 95 \% \mathrm{CI}: 2.99-7.75) . \mathrm{I}^{2}$ test $=85.5 \%$ showed that there was substantial heterogeneity while Egger's test $p=0.124$ depicts that there was no publication bias (Fig. 15) .

\section{Discussion}

This systematic review and meta-analysis explored factors associated with immunization coverage in Ethiopia. To our knowledge, this is the first evidence-based estimate of predictors of immunization coverage among 12-23-month-old children. Maternal educational status, paternal educational status, residence, place of delivery, family size less than four, maternal knowledge on age immunization to be completed, maternal knowledge on the immunization schedule, knowledge on the benefit of immunization, traveling time to the health facility, antenatal care, and TT vaccination was found to be significantly associated with immunization coverage in Ethiopia.

Our study found parental literacy to be a significant predictor of immunization coverage among children age 12-23 months. In this regard, we found that parents that 


\section{A}

Study

ID

OR $(95 \% \mathrm{Cl})$

Weight

Animaw W et al (2014)

Asfaw A et al (2019)

Birhan, Y., et al (2014)

Girmay and Dadi (2019)

Lake et al (2014)

Meleko et al (2018)

Mohamud et al (2014)

Wado et al (2014)

Overall $(\mathrm{I}$-squared $=85.6 \%, p=0.000)$

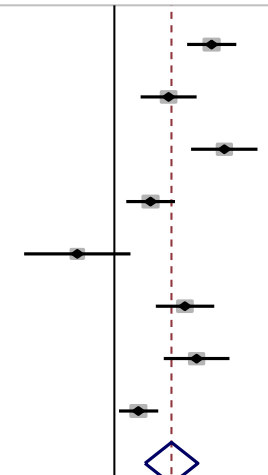

$4.60(3.13,6.77)$

13.33

$2.34(1.51,3.64)$

12.89

$5.62(3.34,9.47)$

12.18

$1.77(1.20,2.59)$

13.35

$0.56(0.24,1.29)$

9.38

$3.03(1.91,4.80)$

12.73

$3.63(2.17,6.08)$

12.23

$1.46(1.07,1.99)$

13.91

$2.45(1.62,3.72)$

100.00

NOTE: Weights are from random effects analysis

Fig. 2 Forest plot depicting the association between mother with formal education and immunization coverage among 12-23 months old children in Ethiopia

had formal education were more likely to fully vaccinate their children compared to their counterpart. Our finding is consistent with other studies that revealed parents who attended formal education were more likely to provide all antigens to their children [54-60]. The current work is also consistent with a study done in Uganda that revealed $46 \%$ of mothers who had primary education fully vaccinated their children while $65 \%$ of those attended secondary education fully vaccinated their children. Thus as maternal education status increases the likelihood of children receiving all antigens increases [61]. Similarly, studies done in Asian countries like India, Vietnam, and Lebanon revealed formally educated mothers are more likely to complete routine immunization than those who did not attend school [62-65]. In contrast, the study done in Ghana revealed that maternal educational status was not significantly associated with full vaccination [55]. The possible reasons that parents with primary and higher education are more likely to utilize information education and communication (IEC) and understand the importance of immunization service [66].

Our study also found that location of residence played a great role in immunization completion among children. Children that live in urban areas were 1.57 times more likely to be fully vaccinated than those in rural areas. Earlier studies that explored the relationship between residence and immunization coverage reported mixed findings [54, 63, 64, 67] . Some studies found a strong relationship between urban residence and immunization while others did not $[55,68]$. The possible explanation for this disparity could be inherent socioeconomic variations that existed among different study settings. Rural Some settings might exist extremely disadvantaged than urban. Disparity in access to health facility and education are common themes which emerge in many studies conducted in urban-rural communities in many countries, including Ethiopia [69, 70].

Furthermore, the place of delivery was found to be a significant predictor of immunization coverage among children age 12-23 months. To this end, women who gave birth at the health facility were 1.86 times more likely to complete all required antigen to their children compared to women who gave birth at home. This finding is consistent with other findings conducted in many parts of the world [59, $62,63,71]$. On the other hand, studies found home delivery as one of the factors for incomplete vaccination $[55,72,73]$. This could be explained by the fact that the first dose (BCG and OPV0) of routine immunization is provided immediately after delivery, which may increase maternal awareness and motivate mothers to complete the sequential doses [74]. Besides, home delivery if taken as a proxy for women's 


\section{B}

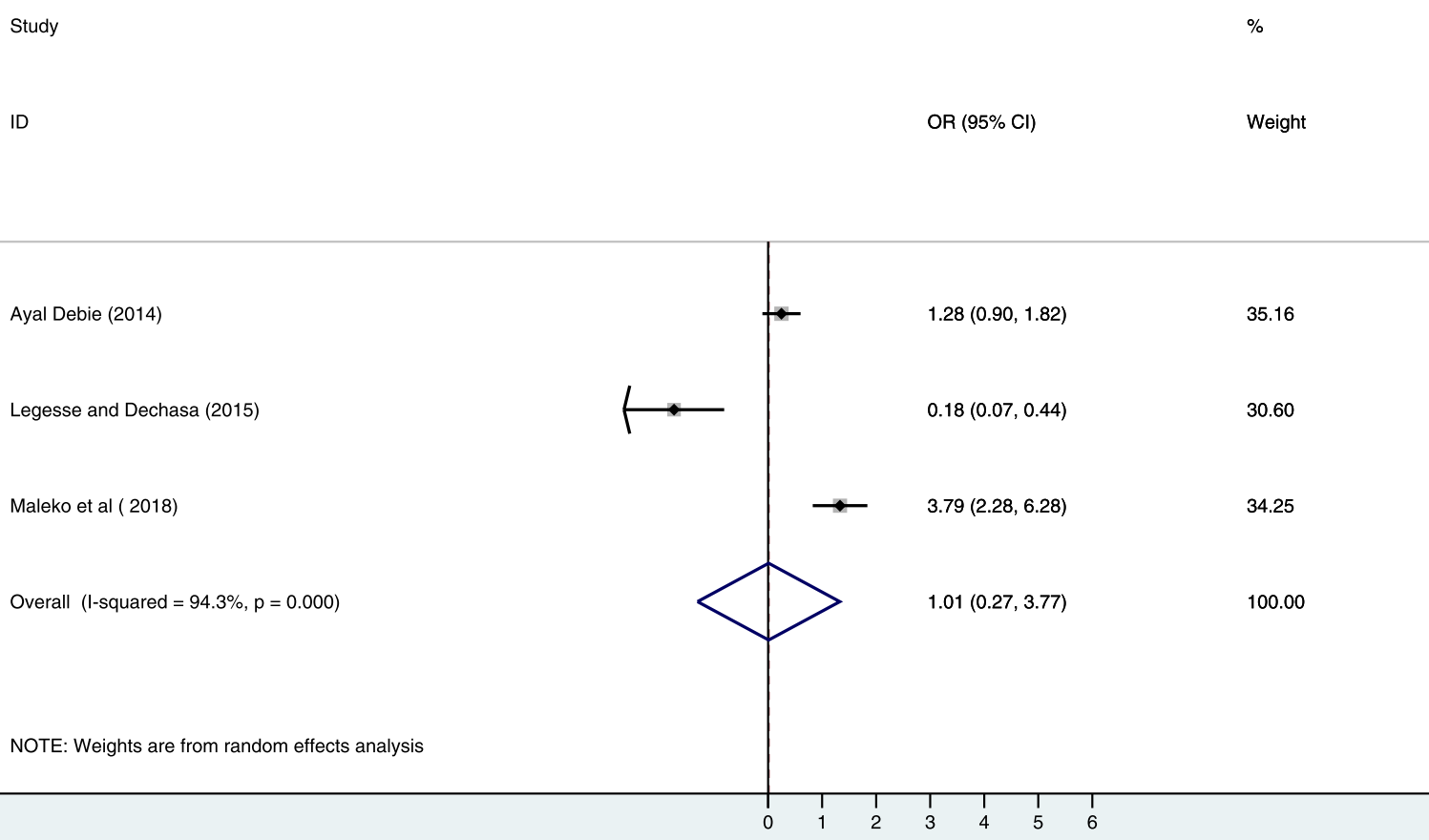

Fig. 3 Forest plot depicting the association between Father with formal Education and immunization coverage among 12-23 months old children in Ethiopia

decision-making autonomy on child's healthcare may reflect its negative influence on child's immunization coverage [75, 76]. Another study also showed that women that had decision-making autonomy were more likely to utilize both institutional delivery, postnatal follow-ups, and child healthcare services [77].

The present study also found that family size was much associated with immunization coverage. Households that had less than four children were more likely to vaccinate their children compared to their counterparts. This finding is in line with studies conducted in Australia [67], India [78], Lebanon [65], Ghana [55] and Brazil [79]. In contrast, the study conducted in Zimbabwe revealed a weak association between household size and immunization coverage [80]. The association between family size and immunization could be explained by the fact that a large family size is likely to hinder maternal capabilities to extend more care to the younger children as well as her mobility to get access to immunization services.

Moreover, in this systematic review and meta-analysis we observed that mothers who are aware of immunization schedule and age the child to complete the immunization program were more likely to complete routine vaccination compared to their counterparts. This finding is in line with a study conducted in Nepal that revealed mothers who had poor knowledge of immunization schedule and age to complete the immunization were four times more likely to complete the immunization program for their children than their counterparts [81]. Likewise, a study done in India showed that $93.5 \%$ of incomplete or partial vaccination was due to lack of knowledge on immunization schedule [82]. This might be due to the fact that mothers who are aware of the immunization schedule were most likely to be well-informed on advantage of immunization and age to complete immunization services, though our findings found no association between knowledge on the benefit of immunization and immunization coverage.

In Ethiopia, distance to the health facility is a major challenge leading to less access to the health services [8]. Our study found traveling time more than a 60 min walk to be a negative contributing factor to immunization coverage. Women whose walk to the health facility was 


\section{C}

Study

ID

OR $(95 \% \mathrm{Cl})$

Weight

\begin{tabular}{|c|c|c|c|}
\hline Ayal D and Bekele T (2014) & 一 & $3.94(1.76,8.82)$ & 14.95 \\
\hline Birhan, Y., et al (2014) & & $0.34(0.17,0.69)$ & 15.63 \\
\hline Kassahun et al (2015) & & $1.47(0.96,2.26)$ & 17.08 \\
\hline Mohamud et al (2014) & - & $2.63(1.85,3.73)$ & 17.41 \\
\hline Tesfaye et al (2018) & $\rightarrow$ & $1.63(1.23,2.15)$ & 17.65 \\
\hline Tessema et al (2019) & $\rightarrow$ & $9.39(6.42,13.75)$ & 17.28 \\
\hline Overall (I-squared $=94.6 \%, p=0.000)$ & & $2.11(1.00,4.45)$ & 100.00 \\
\hline NOTE: Weights are from random effects analysis & & & \\
\hline
\end{tabular}

Fig. 4 Forest plot depicting the association between residence and immunization coverage among 12-23 months old children in Ethiopia

Study

ID

(10)

Animaw $W$ et al (2014)

Ayal D and Bekele T (2014)

Birhan, Y., et al (2014)

Etana and Deressa (2012)

Girmay and Dadi (2019)

Melaku Kindie et al (2018)

Meleko et al (2018)

Mohamud et al (2014)

Tesfaye et al (2018)

Overall (I-squared $=94.6 \%, p=0.000)$

NOTE: Weights are from random effects analysis

\section{D}

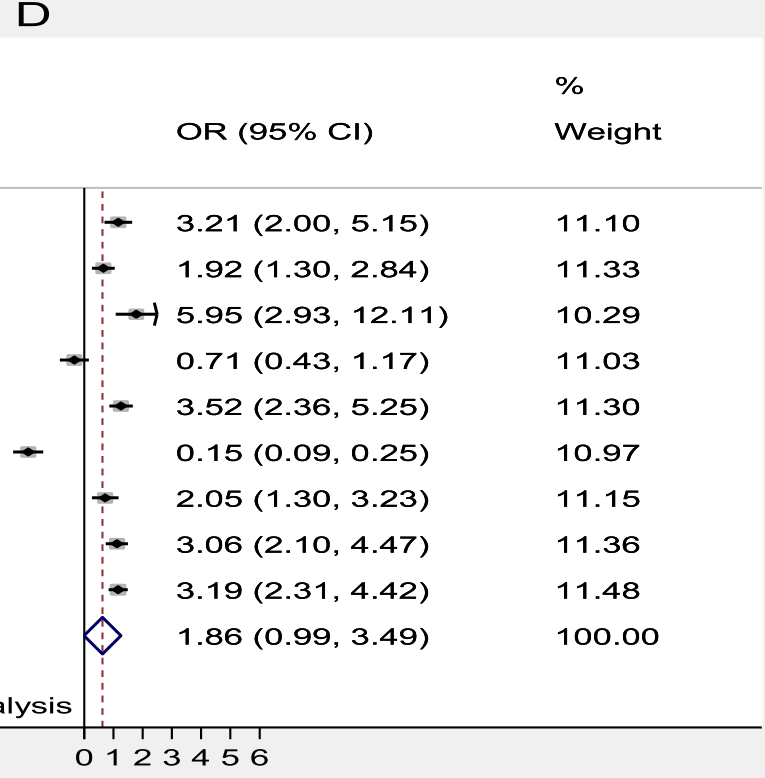

0123456

Fig. 5 Forest plot depicting the association between place of delivery and immunization coverage among 12-23 months old children in Ethiopia 


\section{E}

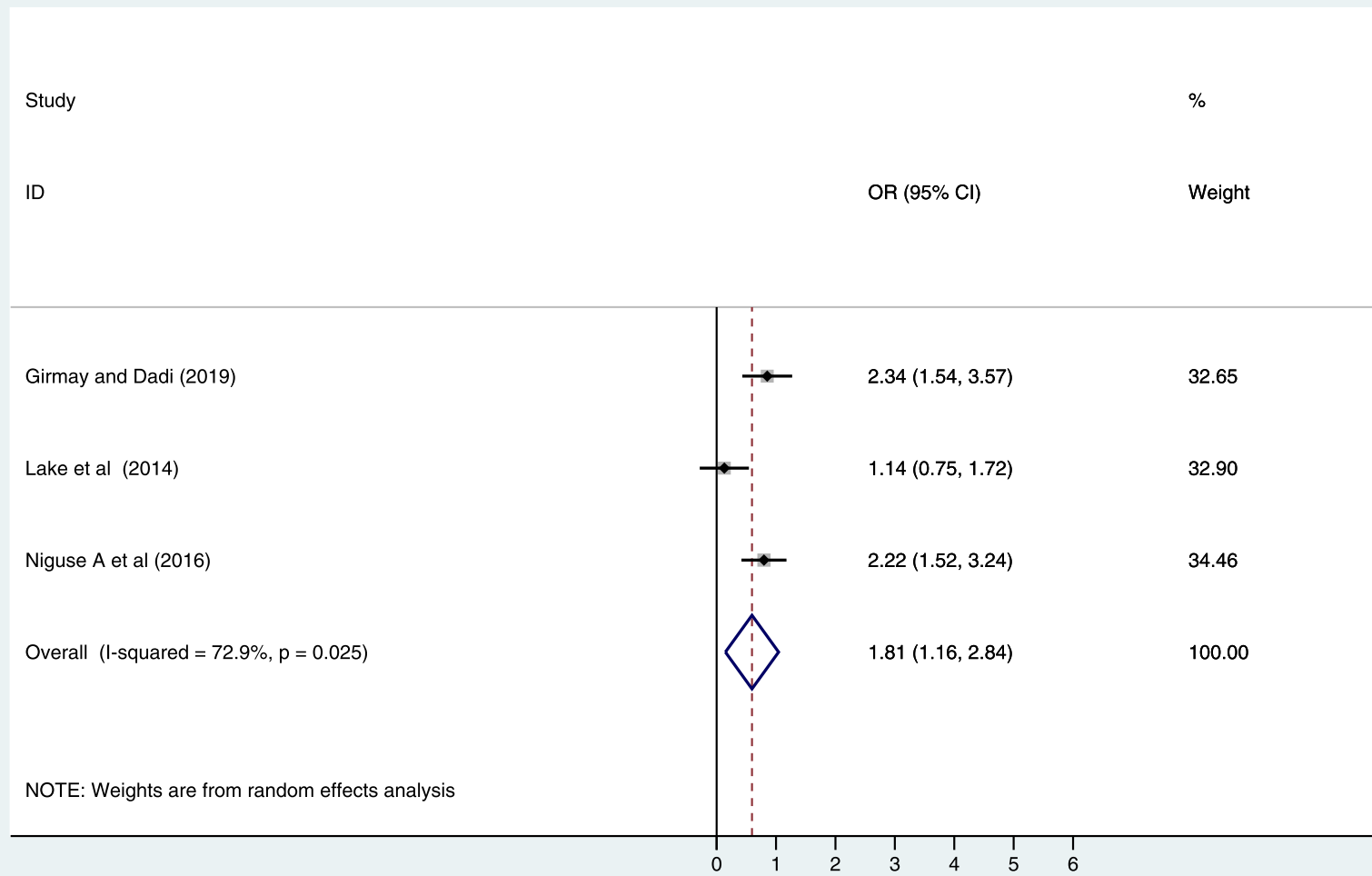

Fig. 6 Forest plot depicting association between Family size and immunization coverage among 12-23 months old children in Ethiopia

less than or equal to 60 min were more likely to vaccinate their children compared to those with a walk above $60 \mathrm{~min}$. This finding is supported by studies done in different parts of the globe [56, 68, 72, 83].

We also found that maternal service utilization to be a predictor of child immunization coverage. Mothers who followed ANC were more likely to complete vaccination for their children compared to their counterparts. This finding is in line with studies conducted in Pakistan [73], Myanmar [84] Indonesia [78], Senegal [59] India [85], and Philippines [86], and a study done in 46 low and middle income countries (LMIC) [87]. Mothers who are following both ANC and PNC are more likely to interact with health care providers and exposed to information about maternal and newborn health services [78]. Similarly, mothers who took TT vaccination were almost five times more likely to complete vaccination for their children compared to those who were not vaccinated. This finding is comparable with a study done in Myanmar [84] that revealed an association between TT vaccination and immunization coverage among children 12-23 months. Maternal service utilization such as ANC and TT vaccinations are found to be proxy indicators that enhanced 19 and 13\% immunization coverage among children aged 12-23 months respectively [88]. Similarly, institutional delivery [89] and a decision made during pregnancy to vaccinate the child also increases the chance of full vaccination [90].

\section{Strengths}

We registred the protocol in Prospero and strictly followed PRISMA guidelines. We have also done a comprehensive literature search and included more factors for this study.

\section{Limitation}

The current work is not without limitations. First, we reviewed only observational studies that cannot be used to infer cause and effect relationship. Community-based studies are also prone to recall bias for infant's vaccination status. Restriction of our search to only English language articles limited the number of articles included in this systematic review and meta-analysis. High heterogeneity was observed from included factors in spite of using the random-effect model. The current work also did not include grey literature/unpublished literature and the potential for publication bias. No study found from some regions of Ethiopia like Benshagul Gumuz. 


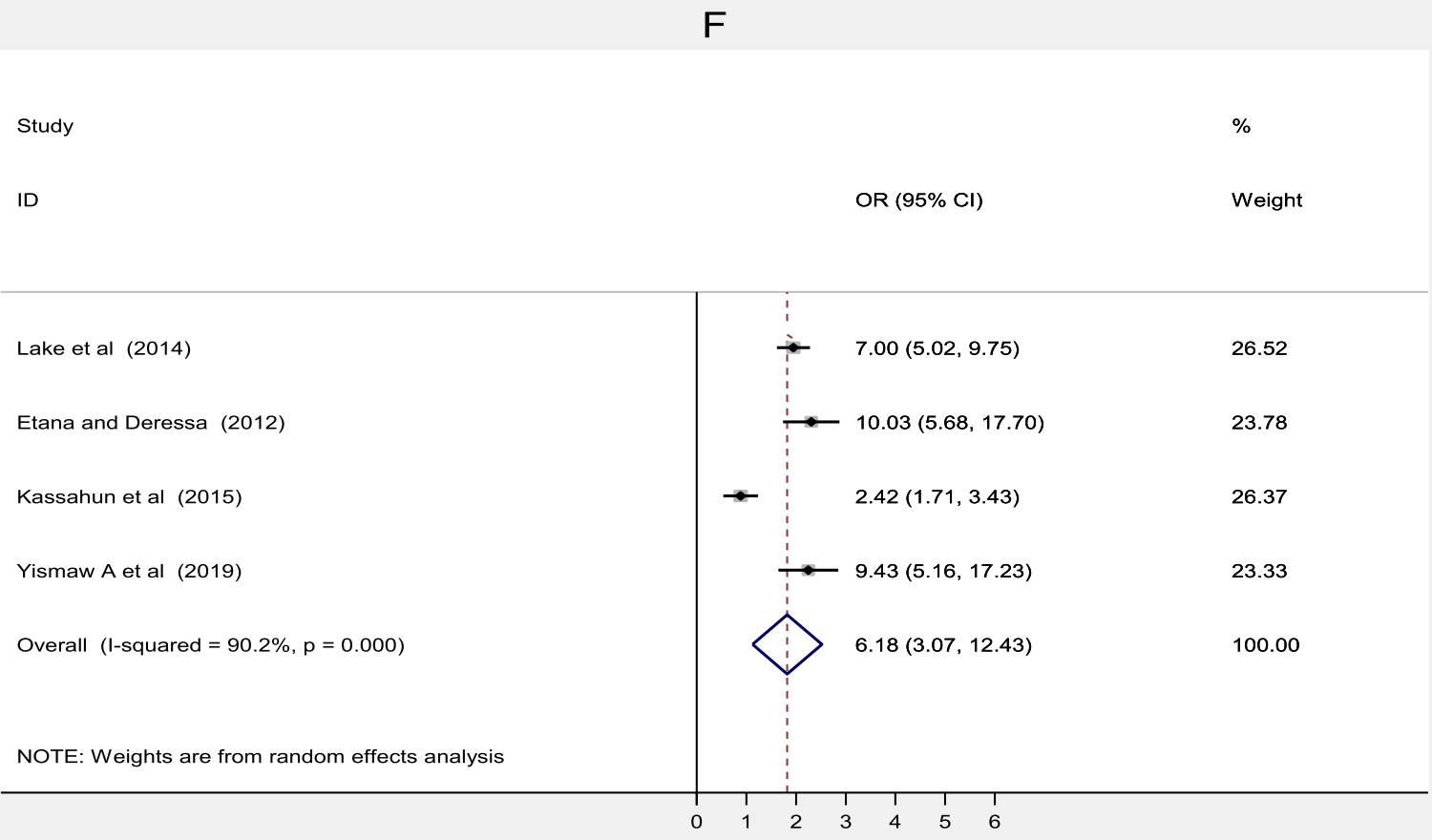

Fig. 7 Forest plot depicting the association between knowledge on age to complete immunization and immunization coverage among $12-23$ months old children in Ethiopia

\section{G}

Study

ID

\section{OR $(95 \% \mathrm{Cl}) \quad$ Weight}

Animaw $W$ et al (2014)

Aregawi $G$ et al (2017)

Asfaw A et al (2019)

Ayal D and Bekele T (2014)

Birhan, Y., et al (2014)

Bizuneh Ayano (2014)

Girmay and Dadi (2019)

Hailu et al (2019)

Lake et al (2014)

Legesse and Dechasa (2015)

Mebrahtom and Birhane (2013)

Tesfaye et al (2018)

Overall (I-squared $=96.2 \%, p=0.000$ )

NOTE: Weights are from random effects analy\$is
$\%$

8.40

8.14

8.32

7.94

8.11

8.45

8.43

8.64

8.55

8.45

8.02

8.55

100.00

\section{$\begin{array}{lllllll}0 & 1 & 2 & 3 & 4 & 5 & 6\end{array}$}

Fig. 8 . Forest plot depicting the association between knowledge on immunization schedule and immunization coverage in Ethiopia 


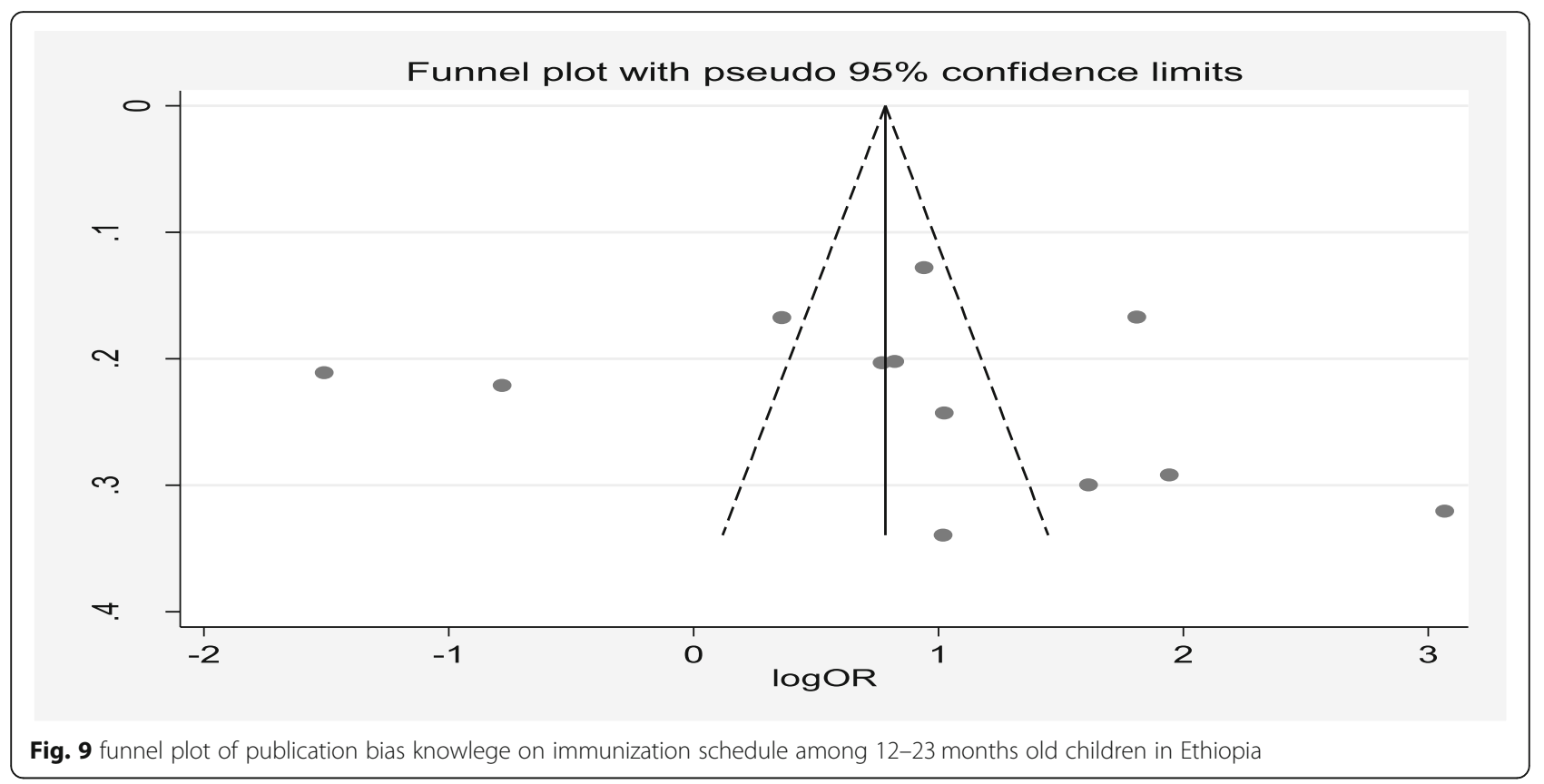

$\mathrm{H}$

Study

ID

OR $(95 \% \mathrm{Cl})$

Weight

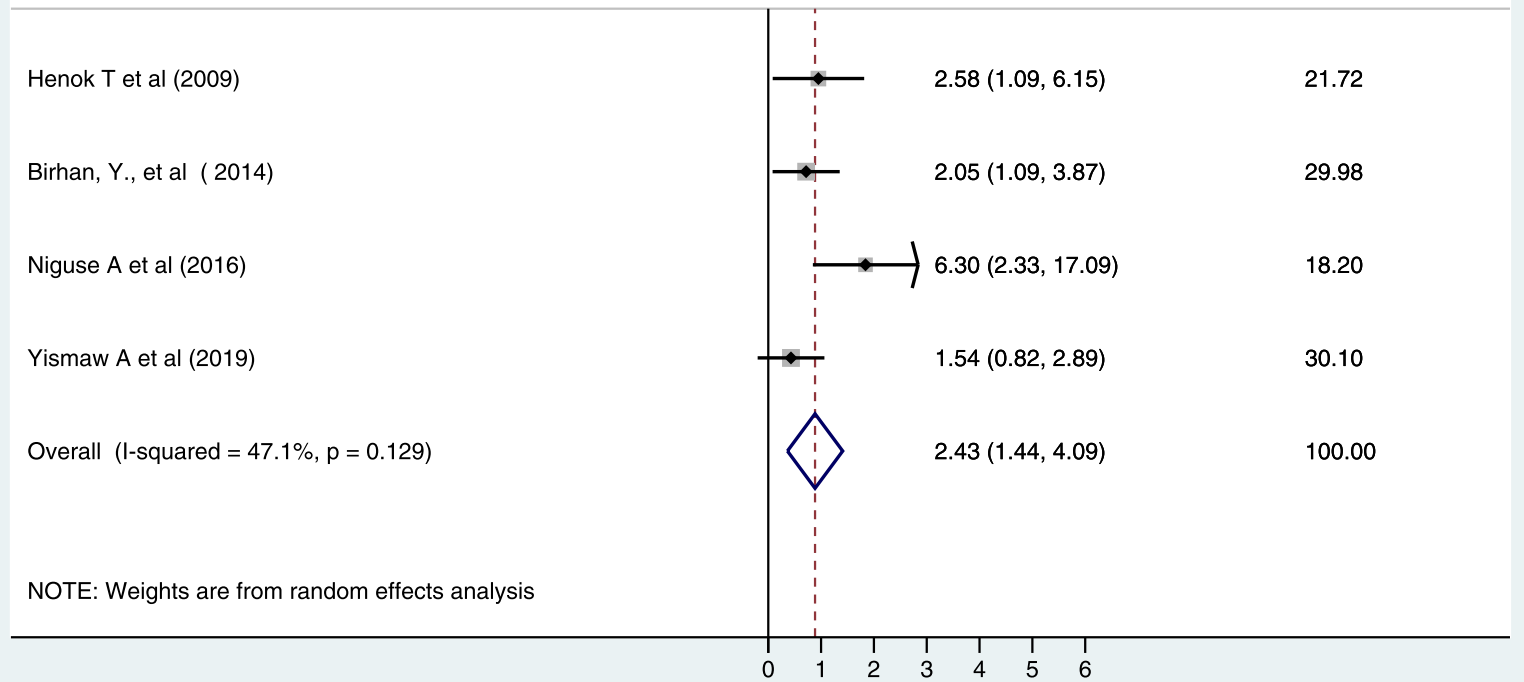

Fig. 10 Forest plot depicting the association between knowledge benefit of immunization and immunization coverage among $12-23$ months old children in Ethiopia 
Study

$\%$
OR $(95 \% \mathrm{Cl}) \quad$ Weight

ID

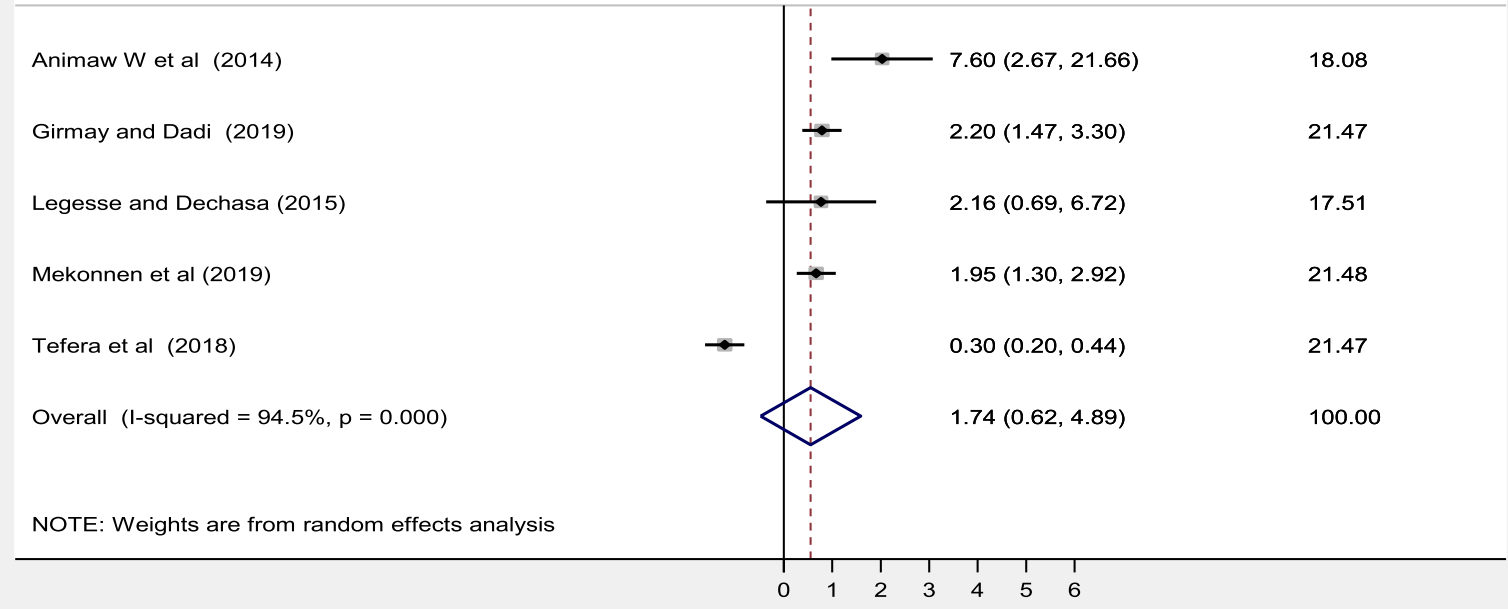

Fig. 11 Forest plot depicting the association between traveling time to the health facility and immunization coverage among 12-23 months old children in Ethiopia

\section{$J$}

Study

ID

Birhan, Y., et al (2014)

Bizuneh Ayano (2014)

Etana and Deressa (2012)

Girmay and Dadi (2019)

Gualu and Dilia (2017)

Hailu et al (2019)

Legesse and Dechasa (2015)

Melaku Kindie et al (2018)

Mebrahtom and Birhane (2013)

Mohammed and Atomsa (2013)

Tefera et al 2018 (2018)

Tesfaye et al (2018)

Overall (I-squared $=95.0 \%, p=0.000$ )

NOTE: Weights are from random effects analy\$is

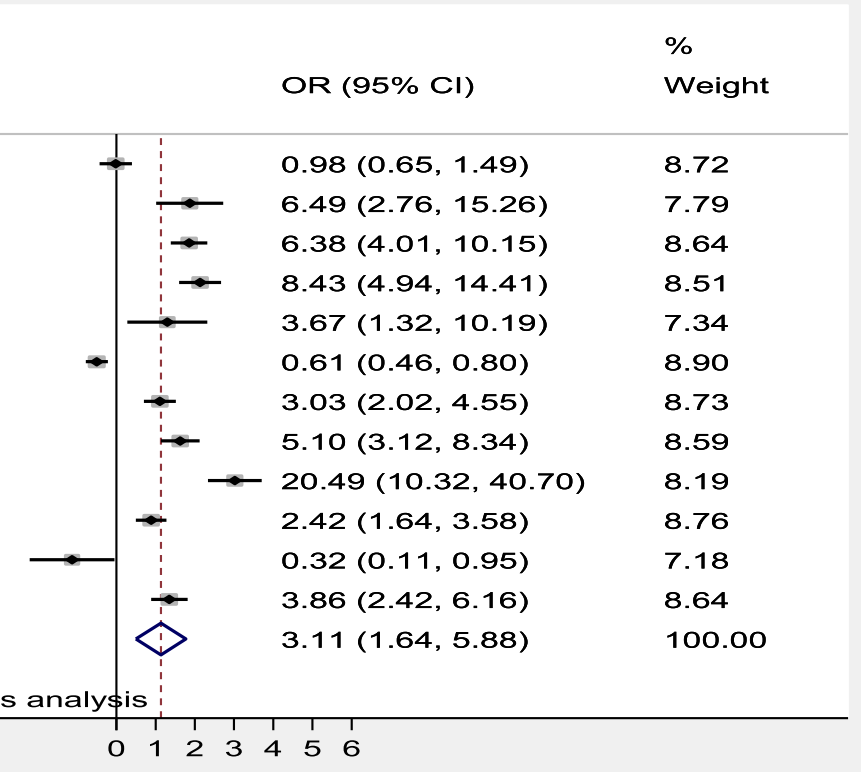

Fig. 12 Forest plot depicting the association between ANC and immunization coverage among 12-23 months old children in Ethiopia 


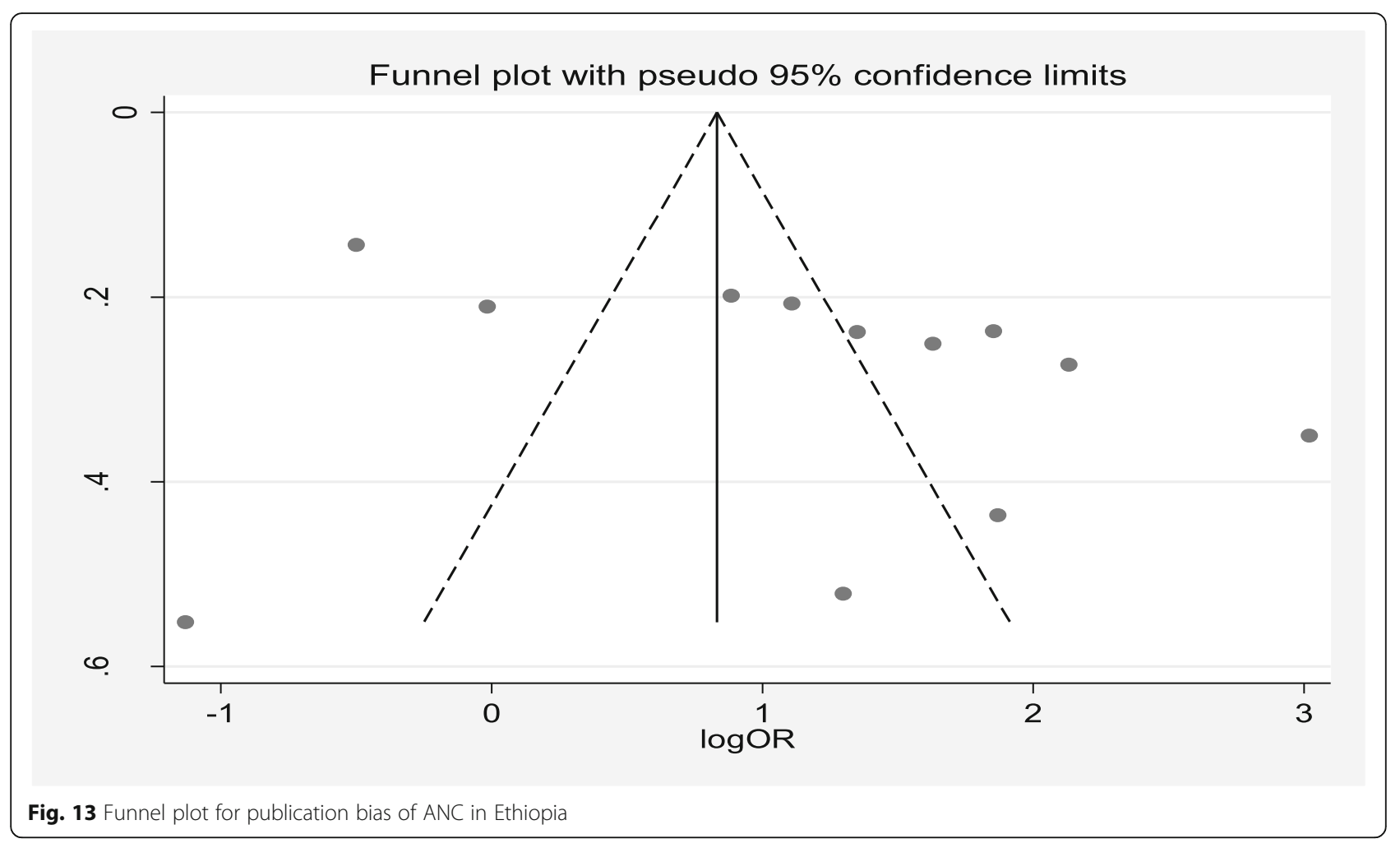

K

Study

ID
OR $(95 \% \mathrm{Cl})$

Weight

\begin{tabular}{|c|c|c|}
\hline Aregawi G et al (2017) & $7.08(3.67,13.65)$ & 19.12 \\
\hline Asfaw A et al (2019) & $2.69(1.73,4.17)$ & 28.91 \\
\hline Mebrahtom and Birhane (2013) & $4.07(2.67,6.22)$ & 29.80 \\
\hline Melaku Kindie et al (2018) & $3.28(1.84,5.85)$ & 22.16 \\
\hline Overall $(I-$ squared $=51.2 \%, p=0.105)$ & $3.83(2.65,5.52)$ & 100.00 \\
\hline NOTE: Weights are from random effects analysis & & \\
\hline
\end{tabular}

Fig. 14 Forest plot depicting the association between PNC and immunization coverage among 12-23 month old children in Ethiopia 
Study

ID

Birhan, Y., et al (2014)

Bizuneh Ayano (2014)

Hailu et al (2014)

Kassahun et al (2015)

Mebrahtom and Birhane (2013)

Mohamud et al (2014)

Overall (I-squared $=85.8 \%, p=0.000)$

OR $(95 \% \mathrm{Cl})$

Weight

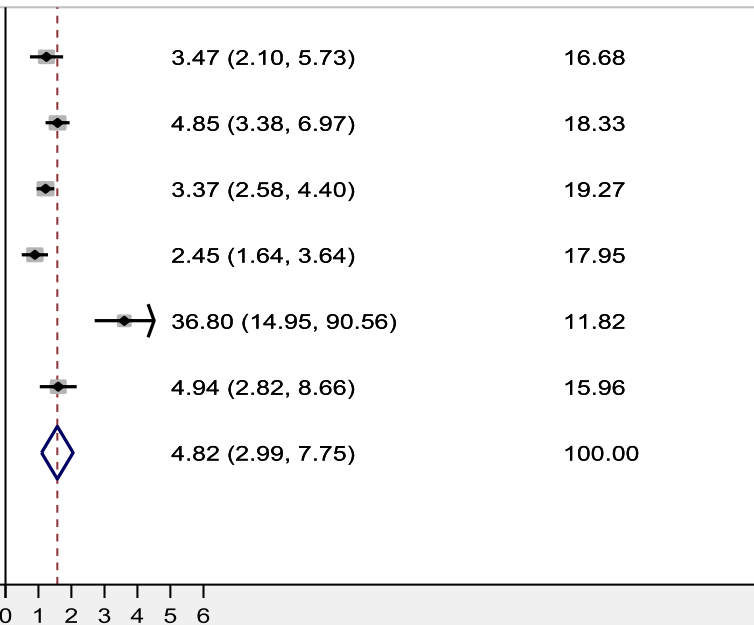

Fig. 15 Forest plot depicting the association between $\Pi$ vaccination and immunization coverage among 12-23 months old children in Ethiopia

\section{Conclusion}

Our findings showed that full immunization among $12-$ 23 months old children are affected by the individual, community, and health service delivery level factors such as literacy, residence, awareness, family size, maternal health services utilization, and proximity of the health facilities were factors associated with full immunization among 12-23 months old children. This implies that there is a need for primary health service expansion and health education to "hard to reach areas" to improve immunization coverage for children aged 12-23 months.

\section{Supplementary Information}

The online version contains supplementary material available at https://doi. org/10.1186/s12889-020-09890-0.

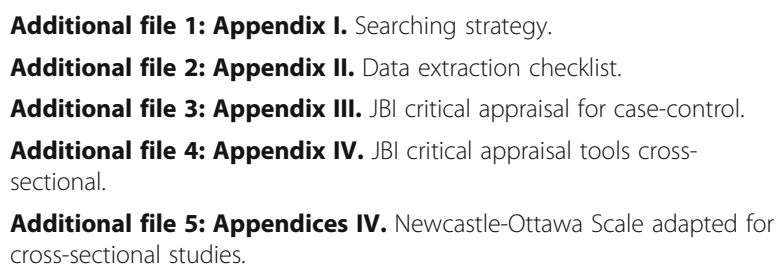

\section{Abbreviations}

BCG: Bacillus Calmette Guerin; Cl: Confidence Interval; DHS: Demographic Health Survey; DPT: Diphtheria Pertussis Tetanus; EDHS: Ethiopia Demographic Health Survey; EPI: Expanded Program of Immunization; HMIS: Health Management Information System; IRIS: Institutional Repository for Information Sharing; JBI: Joanna Briggs Institute; LMIC: Low and Middle Income Countries; PNC: Postnatal Care; PRISMA: Preferred Reporting Items of Systematic Reviews and Meta-Analysis; SDG: Sustainable Development Goals; TT: Tetanus Toxoid; VPD: Vaccine Preventable Diseases; WHO: World Health organization

\section{Acknowledgments}

The authors would like to thank Alan GUTSELL for his assistance copy editing of our manscript. Also would like to express appreciation to the staff of collage of medicine and health science for their support. Lastly, we would like to thank DHS forum for their guidance on DHS data extraction.

\section{Authors' contributions}

TY and AM conceived the study, study selection, preparation of protocol, searching strategy for literature, participated data analysis, interpretation, and manuscript preparation. $\mathrm{OM}, \mathrm{MO}$, and $\mathrm{MA}$ were involved in developing the protocol, searching strategy, critical appraisal, data extraction, while KH prepared and supported the final draft of the manuscript. All authors (TY, $\mathrm{AM}, \mathrm{OM}, \mathrm{MO}, \mathrm{MA}$, and $\mathrm{KH}$ ) were involved in revising and editing the final version of the manuscript. All authors have read and approved the final draft of the manuscript.

\section{Funding}

This meta-analysis has no any funding agencies in government or nongovernment organizations.

\section{Availability of data and materials}

All data generated or analyzed during this systematic review and metaanalysis is included in this article.

\section{Ethics approval and consent to participate}

This systematic review and meta-analysis was done based on already published research which didn't include human subjects. Neither human consents were needed nor institutional review board approval. 


\section{Consent for publication}

Not applicable.

\section{Competing interests}

The author declares there was no competing of interest.

\section{Author details}

'Department of public health, College of Medicine and Health Sciences, Jigjiga University, P.O. BOX 1020, Jigjiga, Ethiopia. ${ }^{2}$ Department of Population and Family Health, Jimma University, Jimma, Ethiopia.

\section{Received: 8 June 2020 Accepted: 15 November 2020}

\section{Published online: 26 November 2020}

\section{References}

1. Wondwossen $L$, et al. Advances in the control of vaccine preventable diseases in Ethiopia. Pan Afr Med J. 2017;27(Suppl 2):1.

2. Organization, W.H. Challenges in global immunization and the global immunization vision and strategy 2006-2015. Wkly Epidemiol Rec. 2006; 81(19):190-5.

3. Organization, W.H., Expanded programme on Immunization (EPI) factsheet 2019: Indonesia. 2019.

4. Lim SS, et al. Tracking progress towards universal childhood immunisation and the impact of global initiatives: a systematic analysis of three-dose diphtheria, tetanus, and pertussis immunisation coverage. Lancet. 2008; 372(9655):2031-46.

5. Bank TW. Estimates Developed by the UN Inter-agency Group for Child Mortality Estimation ( UNICEF, WHO, World Bank, UN DESA Population Division ). 2019; Available from: https://data.worldbank.org/indicator/SH. DYN.MORT.MA?locations=ZG-1W. [cited 2020 May 26].

6. WHO. Child mortality 2011 ; Available from: https://www.who.int/pmnch/ media/press_materials/fs/fs_mdg4_childmortality/en/.[cited 2020 May 26].

7. Walker CLF, et al. Global burden of childhood pneumonia and diarrhoea. Lancet. 2013;381(9875):1405-16.

8. CSA, I., Central statistical agency (CSA)[Ethiopia] and ICF. Ethiopia demographic and health survey, Addis Ababa, Ethiopia and Calverton, Maryland, USA, 2016

9. Organization, W.H. Children: reducing mortality. Fact Sheet. 2016. 2017; Available from: https://scholar.google.com/scholar?hl=en\&as_sdt=0\%2C5 $\& q=$ World +Health+Organization\%3A+Children\%3A+reducing+mortality+ fact+sheet+2017\&btnG=. [cited 2020 May 5].

10. Diouf $\mathrm{K}$, et al. Diarrhoea prevalence in children under five years of age in rural Burundi: an assessment of social and behavioural factors at the household level. Glob Health Action. 2014;7(1):24895.

11. Carvajal-Vélez $L$, et al. Diarrhea management in children under five in subSaharan Africa: does the source of care matter? A Countdown analysis. BMC Public Health. 2016;16(1):830.

12. Organization, W.H. The global action plan to increase supply of pandemic influenza vaccines: report of the fourth meeting of the WHO global action plan of the advisory group, Nha Trang, Viet Nam, 6 May 2010. Geneva: World Health Organization; 2010

13. WHO. Ethiopia Launches Measles Vaccine Second Dose (MCV2) Introduction: Over 3.3 million children will receive the vaccine annually. 2019; Available from: https://www.afro.who.int/news/ethiopia-launches-measles-vaccine-seconddose-mcv2-introduction-over-33-million-children-will.

14. Organization, W.H. World health statistics 2013: a wealth of information on global public health. Geneva: World Health Organization: 2013.

15. Liu L, et al. Global, regional, and national causes of child mortality in 200013, with projections to inform post-2015 priorities: an updated systematic analysis. Lancet. 2015;385(9966):430-40.

16. Hashi A, Kumie A, Gasana J. Prevalence of diarrhoea and associated factors among under-five children in Jigjiga District, Somali region, eastern Ethiopia. Open J Prev Med. 2016;6(10):233-46.

17. Ababa A. Federal democratic republic of Ethiopia ministry of health. Ethiopia: Postnatal Care; 2003.

18. Ethiopia, F.D.R.o. National expanded program on immunization comprehensive multi-year plan 2011-2015. Addis Ababa: Federal Ministry of Health; 2010.

19. Beyene EZ, et al. Factors associated with immunization coverage among children age 12-23 months: the case of zone 3, Afar regional state, Ethiopia. Ethiopian Med J. 2013;51(SUPPL. 1):41-50.
20. Tesfaye TD, Temesgen WA, Kasa AS. Vaccination coverage and associated factors among children aged 12-23 months in Northwest Ethiopia. Hum Vaccin Immunother. 2018;14(10):2348-54.

21. Etana B, Deressa W. Factors associated with complete immunization coverage in children aged 12-23 months in Ambo Woreda, Central Ethiopia. BMC Public Health. 2012;12(1):566.

22. Gualu T, Dilie A. Vaccination coverage and associated factors among children aged 12-23 months in debre markos town, Amhara regional state, Ethiopia. Adv Public Health. 2017;2017.

23. Asfaw $A G$, et al. Determinants of default to fully completion of immunization among children aged 12 to 23 months in South Ethiopia: unmatched case-control study. Pan Afr Med J. 2016;23:100.

24. Organization, W.H. Immunization coverage cluster survey: reference manual. Geneva: World Health Organization; 2005.

25. Moskalewicz A, Oremus M. No clear choice between Newcastle-Ottawa scale and appraisal tool for cross-sectional studies to assess methodological quality in cross-sectional studies of health-related quality of life and breast cancer. J Clin Epidemiol. 2020;120:94-103.

26. Lo CK-L, Mertz D, Loeb M. Newcastle-Ottawa scale: comparing reviewers' to authors' assessments. BMC Med Res Methodol. 2014;14(1):45.

27. Higgins JP, et al. Cochrane handbook for systematic reviews of interventions. Chichester: Wiley; 2019.

28. Song $F$, et al. Asymmetric funnel plots and publication bias in meta-analyses of diagnostic accuracy. Int J Epidemiol. 2002;31(1):88-95.

29. Moher D, Liberati A, Tetzlaff J, Altman DG, The PRISMA Group. Preferred Reporting Items for Systematic Reviews and Meta-Analyses: The PRISMA Statement. PLoS Med. 2009;6(6):e1000097.

30. Animaw W, Taye W, Merdekios B, Tilahun M, Ayele G. Expanded program of immunization coverage and associated factors among children age 12-23 months in Arba Minch town and Zuria District, Southern Ethiopia, 2013. BMC Public Health. 2014;14(1):464.

31. Aregawi $\mathrm{HG}$, et al. Determinants of defaulting from completion of child immunization in Laelay Adiabo District, Tigray region, northern Ethiopia: a case-control study. PLoS One. 2017;12(9):e0185533.

32. Debie A, Taye B. Assessment of fully vaccination coverage and associated factors among children aged 12-23 months in Mecha District, north West Ethiopia: a cross-sectional study. Sci J Public Health. 2014;2(4):342-8.

33. Birhan Y, Tamiso A, Tadele T, Tesfaye F. Predictors of Immunization Defaulting Among Children Age 12-23 Months in Hawassa Zuria District of Southern Ethiopia: Community Based Unmatched Case-Control Study. Int J Public Health Sci. 2014;3(3):7191.

34. Ayano B. Factors affecting fully immunization status of children aged 12-23 months in hosanna town, South Ethiopia. J Preg Child Health. 2015;2(185): 10.4172.

35. Girmay A, Dadi AF. Full immunization coverage and associated factors among children aged 12-23 months in a hard-to-reach areas of Ethiopia. Int J Pediatr. 2019;2019.

36. Hailu S, et al. Low immunization coverage in Wonago district, southern Ethiopia: a community-based cross-sectional study. PLoS One. 2019;14(7): e0220144.

37. Tadesse H, Deribew A, Woldie M. Predictors of defaulting from completion of child immunization in south Ethiopia, May 2008-A case-control study. BMC Public Health. 2009;9(1):150.

38. Kassahun MB, Gashaw AB, Alemayehu ST. Level of immunization coverage and associated factors among children aged 12-23 months in Lay Armachiho District, North Gondar Zone, Northwest Ethiopia: a communitybased cross-sectional study. BMC Res Notes. 2015;8(1):1-10.

39. Legesse E, Dechasa W. An assessment of child immunization coverage and its determinants in Sinana District, Southeast Ethiopia. BMC Pediatr. 2015; 15(1):31.

40. Yenit MK, Gelaw YA, Shiferaw AM. Mothers' health service utilization and attitude were the main predictors of incomplete childhood vaccination in east-Central Ethiopia: a case-control study. Arch Public Health. 2018;76(1):14.

41. Meleko A, Geremew M, Birhanu F. Assessment of child immunization coverage and associated factors with full vaccination among children aged 12-23 months at Mizan Aman town, bench Maji zone, Southwest Ethiopia. Int J Pediatr. 2017;2017:7976587.

42. Mebrahtom S, Birhane Y. Magnitude and determinants of childhood vaccination among pastoral community in Amibara District, Afar regional state, Ethiopia. Res J Med Sci Pub Health. 2013;1(3):22-35. 
43. Mekonnen AG, Bayleyegn AD, Ayele ET. Immunization coverage of 12-23 months old children and its associated factors in Minjar-Shenkora district,Ethiopia: a community-based study. BMC Pediatr. 2019;19(1):1-8.

44. Mohamud AN, et al. Immunization coverage of 12-23 months old children and associated factors in Jigjiga District, Somali National Regional State, Ethiopia. BMC Public Health. 2014;14:865.

45. Negussie A, et al. Factors associated with incomplete childhood immunization in Arbegona district, southern Ethiopia: a case-control study. BMC Public Health. 2015;16(1):27

46. Tefera M, Sharma M. Determinants of immunization among children aged 12-23 months in Ethiopia: a proportional odds model approach. Int J Statistics Med Res. 2015;4(1):140.

47. Tessema F, Kidanne L, Bisrat F, Asres M, Tadesse T, Asress A, Asegdew B, Zeleke S, Bederu N. Child vaccination coverage and dropout rates in pastoral and semi-pastoral regions in Ethiopia: CORE Group Polio Project implementation areas. Ethiop J Health Dev. 2019;33.

48. Wado YD, Afework MF, Hindin MJ. Childhood vaccination in rural southwestern Ethiopia: the nexus with demographic factors and women's autonomy. Pan Afr Med J. 2014;17:9.

49. Yismaw AE, et al. Incomplete childhood vaccination and associated factors among children aged 12-23 months in Gondar city administration, northwest, Ethiopia 2018. BMC Res Notes. 2019;12(1):241.

50. Lake $\mathrm{M}$, et al. Factors for low routine immunization performance; a community based cross sectional study in Dessie town, south Wollo zone, Ethiopia, 2014. Adv Appl Sci. 2016;1:7-17.

51. Mohammed H, Atomsa A. Assessment of child immunization coverage and associated factors in Oromia regional state, eastern Ethiopia. Sci Technol Arts Res J. 2013;2(1):36-41.

52. Lakew $Y$, Bekele A, Biadgilign S. Factors influencing full immunization coverage among 12-23 months of age children in Ethiopia: evidence from the national demographic and health survey in 2011. BMC Public Health. 2015;15:728.

53. Ebrahim T, Beyene W. Childhood immunization coverage in tehulederie district, northeast of Ethiopia: a community based cross sectional study. Int J Curr Res. 2015;7(9):20234-40.

54. Wiysonge CS, Uthman OA, Ndumbe PM, Hussey GD. Individual and contextual factors associated with low childhood immunization coverage in sub-Saharan Africa: a multilevel analysis. PLoS One. 2012;7(5):e37905.

55. Moran EB, et al. Socioeconomic characteristics associated with the introduction of new vaccines and full childhood vaccination in Ghana, 2014. Vaccine. 2020:38(14):2937-2942.

56. Ekouevi DK, et al. Incomplete immunization among children aged 12-23 months in Togo: a multilevel analysis of individual and contextual factors. BMC Public Health. 2018;18(1):952.

57. Adeloye $\mathrm{D}$, et al. Coverage and determinants of childhood immunization in Nigeria: a systematic review and meta-analysis. Vaccine. 2017;35(22):2871-81.

58. Chiabi $A$, et al. Vaccination of infants aged 0 to 11 months at the Yaounde Gynaeco-obstetric and pediatric hospital in Cameroon: how complete and how timely? BMC Pediatr. 2017;17(1):206.

59. Mbengue MAS, et al. Determinants of complete immunization among senegalese children aged 12-23 months: evidence from the demographic and health survey. BMC Public Health. 2017;17(1):630.

60. Adenike OB, Adejumoke J, Olufunmi O, Ridwan O. Maternal characteristics and immunization status of children in North Central of Nigeria. Pan Afr Med J. 2017;26.

61. Nankabirwa $V$, et al. Maternal education is associated with vaccination status of infants less than 6 months in eastern Uganda: a cohort study. BMC Pediatr. 2010;10(1):92.

62. Pandey $\mathrm{S}$, et al. Socio-demographic determinants of childhood immunization coverage in rural population of Bhojpur district of Bihar, India. J Fam Med Prim Care. 2019;8(7):2484.

63. An DTM, et al. Timely immunization completion among children in Vietnam from 2000 to 2011: a multilevel analysis of individual and contextual factors. Glob Health Action. 2016;9(1):29189.

64. Phukan RK, Barman MP, Mahanta J. Factors associated with immunization coverage of children in Assam, India: over the first year of life. J Trop Pediatr. 2009;55(4):249-52.

65. Kmeid $M$, et al. Vaccine coverage for Lebanese citizens and Syrian refugees in Lebanon. Int Health. 2019;11(6):568-79.

66. Vikram K, Desai S, Vanneman R. Maternal education and child mortality: Exploring the pathways of influence; 2010.
67. Homel J, Edwards B. Factors associated with delayed infant immunization in a nationally representative cohort study. Child Care Health Dev. 2018;44(4):583-91.

68. Crocker-Buque T, et al. Immunization, urbanization and slums-a systematic review of factors and interventions. BMC Public Health. 2017;17(1):556.

69. Okwaraji YB, et al. Effect of geographical access to health facilities on child mortality in rural Ethiopia: a community based cross sectional study. PLoS One. 2012;7(3):e33564.

70. Nigussie M, Mariam DH, Mitike G. Assessment of safe delivery service utilization among women of childbearing age in North Gondar zone, north West Ethiopia. Ethiop J Health Dev. 2004;18(3):145-52.

71. Antai D. Inequitable childhood immunization uptake in Nigeria: a multilevel analysis of individual and contextual determinants. BMC Infect Dis. 2009;9(1):181.

72. Jani JV, et al. Risk factors for incomplete vaccination and missed opportunity for immunization in rural Mozambique. BMC Public Health. 2008;8(1):161.

73. Bugvi AS, et al. Factors associated with non-utilization of child immunization in Pakistan: evidence from the demographic and health survey 2006-07. BMC Public Health. 2014;14(1):232.

74. Kumar D, Aggarwal A, Gomber S. Immunization status of children admitted to a tertiary-care hospital of north India: reasons for partial immunization or non-immunization. J Health, Popul Nutr. 2010;28(3):300.

75. Ghose B, Feng D, Tang S, Yaya S, He Z, Udenigwe O, Ghosh S, Feng Z. Women's decision-making autonomy and utilization of maternal healthcare services: results from the Bangladesh Demographic and Health Survey. BMJ Open. 2017;7(9).

76. Wanaka S, et al. Maternal delays for institutional delivery and associated factors among postnatal mothers at public health facilities of Gamo zone, Southern Ethiopia. Int J Women's Health. 2020;12:127.

77. Nigatu AM, Gelaye KA. Factors associated with the preference of institutional delivery after antenatal care attendance in Northwest Ethiopia. BMC Health Serv Res. 2019;19(1):810.

78. Herliana P, Douiri A. Determinants of immunisation coverage of children aged 12-59 months in Indonesia: a cross-sectional study. BMJ Open. 2017; 7(12):e015790.

79. Tertuliano GC, Stein AT. Immunization delay determinants: a study in a place attended by family health strategy. Cien Saude Colet. 2011;16(2):523-30.

80. Rossi R. Do maternal living arrangements influence the vaccination status of children age 12-23 months? A data analysis of demographic health surveys2010-11 from Zimbabwe. PLoS One. 2015;10(7):e0132357.

81. Shrestha $\mathrm{S}$, et al. Predictors of incompletion of immunization among children residing in the slums of Kathmandu valley, Nepal: a case-control study. BMC Public Health. 2016;16(1):970.

82. Trivedi $\mathrm{R}$, et al. Coverage evaluation of primary immunization and the associated determinants in an urban slum of Rewa. Indian J Community Health. 2014;26(1):37-40

83. Mugali RR, et al. Improving immunization in Afghanistan: results from a cross-sectional community-based survey to assess routine immunization coverage. BMC Public Health. 2017;17(1):290.

84. Nozaki I, Hachiya M, Kitamura T. Factors influencing basic vaccination coverage in Myanmar: secondary analysis of 2015 Myanmar demographic and health survey data. BMC Public Health. 2019;19(1):242.

85. Partha D, Bhattacharya BN. Determinants of child immunization in four lessdeveloped states of North India. J Child Health Care. 2002;6(1):34-50.

86. Bondy JN, et al. Identifying the determinants of childhood immunization in the Philippines. Vaccine. 2009;27(1):169-75.

87. Hajizadeh M. Socioeconomic inequalities in child vaccination in low/middleincome countries: what accounts for the differences? J Epidemiol Community Health. 2018;72(8):719-25.

88. Dixit P, Dwivedi LK, Ram F. Strategies to improve child immunization via antenatal care visits in India: a propensity score matching analysis. PLoS One. 2013;8(6):e66175.

89. Acharya P, Kismul H, Mapatano MA, Hatløy A. Individual-and communitylevel determinants of child immunization in the Democratic Republic ofCongo: a multilevel analysis. PloS One. 2018;13(8):e0202742.

90. Danchin $\mathrm{MH}$, et al. Vaccine decision-making begins in pregnancy: correlation between vaccine concerns, intentions and maternal vaccination with subsequent childhood vaccine uptake. Vaccine. 2018;36(44):6473-9.

\section{Publisher's Note}

Springer Nature remains neutral with regard to jurisdictional claims in published maps and institutional affiliations. 